\title{
Global Well-Posedness and Global Attractor for Two-dimensional Zakharov-Kuznetsov Equation
}

\author{
Minjie Shan * \\ School of Mathematical Sciences, Peking University, Beijing 100871, P.R. China \\ Emails: smj@pku.edu.cn
}

June 14, 2021

\begin{abstract}
The initial value problem for two-dimensional Zakharov-Kuznetsov equation is shown to be globally well-posed in $H^{s}\left(\mathbb{R}^{2}\right)$ for all $\frac{5}{7}<s<1$ via using $I$-method in the context of atomic spaces. By means of the increment of modified energy, the exsitence of global attractor for weakly damped, forced Zakharov-Kuznetsov equation is also established in $H^{s}\left(\mathbb{R}^{2}\right)$ for $\frac{10}{11}<s<1$.
\end{abstract}

Keywords: Zakharov-Kuznetsov equation, Global well-posedness, Global attractor, I-method, Atomic spaces.

\section{Introduction and Main Results}

We consider the Cauchy problem for the symmetrized two-dimensional Zakharov-Kuznetsov (ZK) equation

$$
\left\{\begin{array}{l}
u_{t}+\left(\partial_{x}^{3}+\partial_{y}^{3}\right) u+\left(\partial_{x}+\partial_{y}\right) u^{2}=0, \quad(x, y) \in \mathbb{R}^{2}, t \geqslant 0 \\
u(x, y, 0)=u_{0}(x, y) \in H^{s}\left(\mathbb{R}^{2}\right),
\end{array}\right.
$$

where $u=u(x, y, t)$ is a real-valued function.

The ZK equation was initially decuded as a model of nonlinear unidirectional ionacoustic wave propagation in a magnetized plasma by Zakharov and Kuznetsov [16]. It may be treated as a higher dimensional generalization of the Korteweg-de Vries (KdV) equation. For more details we refer to the papers [17, 18] about the two-dimensional ZK equation appearing here in physical circumstances.

\footnotetext{
${ }^{*}$ Corresponding author. The author was supported by CSC, grant 201606010025
} 
Eventhough the ZK equation is not completely integrable, there still exists two conserved quantities for the flow of ZK,

$$
M(u)(t)=\int_{\mathbb{R}^{2}} u^{2}(x, y, t) d x d y=\int_{\mathbb{R}^{2}} u_{0}^{2}(x, y) d x d y=M\left(u_{0}\right)
$$

and

$$
E(u)(t)=\int_{\mathbb{R}^{2}} \frac{1}{2}|\nabla u|^{2}-\frac{1}{2} \partial_{x} u \partial_{y} u-\frac{1}{3} u^{3} d x d y=E\left(u_{0}\right) .
$$

Faminskii [6] firstly obtained the local well-posedness for the two-dimensional ZK equation in the energy space $H^{1}\left(\mathbb{R}^{2}\right)$ by making use of local smoothing effects together with a maximal function estimate for the linearized equation. This method was inspired by the one given by Kenig, Ponce and Vega [12] when dealing with the local wellposedness for $\mathrm{KdV}$ equation. With the help of the $L^{2}$ and $H^{1}$ conservation laws, he proved the global well-posedness for ZK equation additionally. Following this idea, Linares and Pastor [19] optimized the proof of Faminskii to show local well-posedness in $H^{s}\left(\mathbb{R}^{2}\right)$ for $s>\frac{3}{4}$. Grünrock and Herr [9] along with Molinet and Pilod [21] proved the local well-posedness in a larger data spaces $H^{s}\left(\mathbb{R}^{2}\right)$ for $s>\frac{1}{2}$ by taking advantage of the Fourier restriction norm method and one kind of sharp Strichartz estimates. On the basis of the method of Grünrock and Herr, in [25] we recently improved the local well-posedness result to $B_{2,1}^{\frac{1}{2}}\left(\mathbb{R}^{2}\right)$ via applying frequency decomposion as well as atomic spaces introduced by Koch and Tataru. Actually, it is L. Molinet and D. Pilod who originality applied this crucial tool of atomic spaces to ZK equation. They obtained the global well-posedness for ZK equation in $H^{s}\left(\mathbb{R}^{3}\right)$ for $s>1$ by using the conservation laws, doubling time, the argument of Bourgain (see [2]) and atomic spaces. What's more, as the other part of the same paper [25] we utilized $I$-method to prove global well-posedness in $H^{s}\left(\mathbb{R}^{2}\right)$ for $\frac{11}{13}<s<1$.

As mentioned above, ZK equation is an asymptotic description of the propagation of nonlinear ion-acoustic wave in a magnetized plasma. However, one cannot totally neglect external excitation and energy dissipation mechanism in reality (see [1], 23] and [29]). Therefore, we would like to consider the following Zakharov-Kuznetsov equation with weak dissipation and forcing terms

$$
\left\{\begin{array}{l}
u_{t}+\left(\partial_{x}^{3}+\partial_{y}^{3}\right) u+\left(\partial_{x}+\partial_{y}\right) u^{2}+\gamma u=f, \quad(x, y) \in \mathbb{R}^{2}, t \geqslant 0 \\
u(x, y, 0)=u_{0}(x, y) \in H^{s}\left(\mathbb{R}^{2}\right),
\end{array}\right.
$$

where the damping parameter $\gamma$ is a positive constant and the external forcing term $f \in H^{1}\left(\mathbb{R}^{2}\right)$ is independent of $t$. 
Global attractor is a bounded subset which is invariant by the flow and attracts all trajectories when $t$ approaches to $+\infty$ under the corresponding topology. Moise and Rosa studied the regularity of the global attractor of a weakly damped, forced KdV equation in [20. While Haraux [11] and Goubet [8] proved the asymptotic smoothing effect for dissipative wave equation and nonlinear Schrödinger eqution respectively. As we know that the conservation laws will become invalid in low regularity situation, Colliander, Keel, Staffilani, Takaoka and Tao [3, 4] developed a theory of almost conserved quantities starting with energy in order to extend the local solutions globally in time for dispersive equations. Applying I-method to weakly damped, forced KdV equation, Tsugawa [27] proved the existence of global attractor on Sobolev spaces of negative index. Prashant 24] lately showed an analogue below energy spaces for $\mathrm{mKdV}$ equation.

In this article, we refine the global well-posedness for ZK equation through pushing our previous result to $\frac{5}{7}<s$. The new ingredient here is a closer investigation into how to use the symmetrization and atomic space to acquire a better control of the modified energy. Whereafter the increment of the modified energy helps us gain the existence of global weak attractor below $H^{1}$ for weakly damped, forced ZK equation. The advantage of $U^{2}, V^{2}$ spaces is to obtain sharp estimates in the time variable, which makes the proof simpler than when we use $X^{s, 1 / 2+}$. For instance, as regards local well-posedness for ZK equation in $H^{\frac{1}{2}}\left(\mathbb{R}^{2}\right)$, to some extent it corresponds to the non-admissible endpoint Strichartz estimates. However this can be compensated partly by the decay brought from utilizing atomic space. Especially, the use of $U^{2}, V^{2}$ spaces will assist us to study the problem on the $2 \mathrm{D}$ torus or the $\mathbb{R} \times \mathbb{T}$, which may be nice as a future problem (see also [21] for ZK equation on $\mathbb{R} \times \mathbb{T}$ ).

We now state the main results of this paper.

Theorem 1.1. The initial value problem (1.1) is globally well-posed in $H^{s}\left(\mathbb{R}^{2}\right)$ for $\frac{5}{7}<$ $s<1$.

Remark 1. The increment in $E\left(I_{N} u\right)$ on the right hand of $(\underline{3.16})$ is $N^{-\frac{1}{2}}$. One could repeat the almost conservation law argument to prove global well-posedness of (1.1) for all $\frac{1}{2}<s<1$ upon gaining $N^{-1}$.

Theorem 1.2. Let $\frac{10}{11}<s<1$. Then, there exists a semigroup $A(t)$ and maps $M_{1}$ and $M_{2}$ such that $A(t) u_{0}$ is the unique solution of (1.4) satisfying

$$
\begin{array}{r}
A(t) u_{0}=M_{1}(t) u_{0}+M_{2}(t) u_{0} \\
\sup _{t>T_{1}}\left\|M_{1}(t) u_{0}\right\|_{H^{1}}<K
\end{array}
$$

and for $t>T_{1}$

$$
\left\|M_{2}(t) u_{0}\right\|_{H^{s}}<K e^{-\gamma\left(t-T_{1}\right)}
$$


where $T_{1}$ depends on $\left\|u_{0}\right\|_{H^{s}},\|f\|_{H^{1}}$ and $\gamma$, the constant $K$ depends only on $\|f\|_{H^{1}}$ and $\gamma$.

From this theorem, we know that $M_{1}(t)$ is a bounded mapping and $M_{2}(t)$ converges uniformly to 0 in $H^{s}$. It means that the semigroup $A(t)$ is asymptotically compact in the sense of weak topology. Therefore we gain the existence of the global attractor in $H^{s}$ from Theorem 1.1.1 in [26].

Corollary 1.3. The global weak attractor for (1.4) exisits in $H^{s}$ for $\frac{10}{11}<s<1$.

Note that

$$
\begin{aligned}
\partial_{t} \int u^{2} & =-2 \int u\left(\partial_{x}^{3}+\partial_{y}^{3}\right) u-2 \int u\left(\partial_{x}+\partial_{y}\right) u^{2}-2 \gamma \int u^{2}+2 \int f u \\
& =-2 \gamma \int u^{2}+2 \int f u
\end{aligned}
$$

Setting $h(t)=e^{2 \gamma t}\|u\|_{L^{2}}^{2}$ and using Cauchy-Schwarz inequality in the last term, one can obtain

$$
h^{\prime}(t) \leqslant 2 e^{\gamma t}\|f\|_{L^{2}} \sqrt{h(t)}
$$

This implies that

$$
\partial_{t} \sqrt{h(t)} \leqslant e^{\gamma t}\|f\|_{L^{2}}
$$

Hence, we have

$$
\|u\|_{L^{2}} \leqslant e^{-\gamma t}\left\|u_{0}\right\|_{L^{2}}+\gamma^{-1}\|f\|_{L^{2}} .
$$

Remark 2. For the modified energy, we expect a similar exponential decay as (1.8) (see Proposition 4.4).

Remark 3. Yang [30] upgrated Tsugawa's result through lowering $-\frac{3}{8}$ to $-\frac{1}{2}$ which can be seen as the critical Sobolev index for KdV. However, for ZK equation and mKdV equation it is not easy to find good cancellation of resonant parts as Schrödinger equation (see [5]). Concerning the existence of global attrator, there still are much work to do for these two kinds of equations.

Organization of the paper. In Section 2, we recall some useful propositions and estimates about $U^{p}$ and $V^{p}$. Then we prove global well-posedness by using $I$-method in atomic space in Section 3. Finally, Section 4 is devoted to a prior estimate and the proof of Theorem 1.2.

We now list notations used throughout this paper. Let $c<1, C>3$ denote universal constants. The notation $c+$ stands for $c+\epsilon$ some $0<\epsilon \ll 1$. Similarly, we shall write $c-=c-\epsilon$. We put $\langle a\rangle=\left(1+a^{2}\right)^{\frac{1}{2}}$ for $a \in \mathbb{R}$ and fix a smooth cut-off function 
$\chi \in C_{0}^{\infty}([-2,2])$ satisfing $\chi$ is even, nonnegative, and $\chi=1$ on $[-1,1]$. We denote spatial variables by $x, y$ and their dual Fourier variables by $\xi, \eta$. As usual, $\tau$ is the dual variable of the time $t$. Let $\tilde{f}$ denote the Fourier transform of $f$ in both time and spatial variables. Let $\widehat{f}$ denote its Fourier transform only in space or in time. For $s \in \mathbb{R}, I_{x}^{s}$ and $I_{y}^{s}$ denote the one-dimensional Riesz-potential operators of order $-s$ with respect to spatial variable $x$ and $y$. We also write $\zeta=(\xi, \eta), \lambda=(\xi, \eta, \tau)$ and $\mu=\tau-\xi^{3}-\eta^{3}$ for brevity. We will make frequent use of the capital letters $N, M, N_{1}, N_{2}$ and $N_{3}$ which denote dyadic numbers and we write $\sum_{N \geqslant 1} a_{N}=\sum_{n \in \mathbb{N}} a_{2^{n}}, \sum_{N \geqslant M} a_{N}=\sum_{n \in \mathbb{N} ; 2^{n} \geqslant M} a_{2^{n}}$ for dyadic summations.

\section{$2 \quad$ Function spaces and estimates}

In this section we introduce some properties of $U^{p}$ and $V^{p}$ spaces (see [10, 13, 14, 15]) which is another powful tool to handle low regularity well-posedness for dispersive equations.

Let $1 \leqslant p<\infty$ and $\mathcal{Z}$ be the set of finite partitions $-\infty=t_{0}<t_{1}<\ldots<t_{K-1}<$ $t_{K}=\infty$.

For any $\left\{t_{k}\right\}_{k=0}^{K} \subset \mathcal{Z}$ and $\left\{\phi_{k}\right\}_{k=0}^{K-1} \in L^{2}$ with $\sum_{k=0}^{K-1}\left\|\phi_{k}\right\|_{2}^{p}=1, \phi_{0}=0$. We call the function $a: \mathbb{R} \rightarrow L^{2}$ given by

$$
a=\sum_{k=1}^{K} 1_{\left[t_{k-1}, t_{k}\right)} \phi_{k-1}
$$

a $U^{p}$-atom. The atomic space is

$$
U^{p}=\left\{u=\sum_{j=1}^{\infty} \lambda_{j} a_{j}: a_{j} U^{p}-\text { atom, } \lambda_{j} \in \mathbb{C}, \sum_{j=1}^{\infty}\left|\lambda_{j}\right|<\infty\right\}
$$

endowed with the norm

$$
\|u\|_{U^{p}}=\inf \left\{\sum_{j=1}^{\infty}\left|\lambda_{j}\right|: u=\sum_{j=1}^{\infty} \lambda_{j} a_{j}, a_{j} U^{p}-\text { atom, } \lambda_{j} \in \mathbb{C}\right\} .
$$

$V^{p}$ is the normed space of all functions $v: \mathbb{R} \rightarrow L^{2}$ such that $\lim _{t \rightarrow \pm \infty} v(t)$ exist and for which the norm

$$
\|v\|_{V^{p}}=\sup _{\left\{t_{k}\right\}_{k=0}^{K} \in \mathcal{Z}}\left(\sum_{k=1}^{K}\left\|v\left(t_{k}\right)-v\left(t_{k-1}\right)\right\|_{L^{2}}^{p}\right)^{\frac{1}{p}}
$$

is finite, where we use the convention that $v(-\infty)=\lim _{t \rightarrow-\infty} v(t)$ and $v(\infty)=0$. We denote $v \in V_{-}^{p}$ when $v(-\cdot) \in V^{p}$. Moreover, we define the closed subspace $V_{r c}^{p}\left(V_{-, r c}^{p}\right)$ of all right continuous functions in $V^{p}\left(V_{-}^{p}\right)$. 
The unitary operator $e^{t S}: L^{2} \rightarrow L^{2}$ is defined to be the Fourier multiplier

$$
\widehat{e^{t S} u_{0}}(\xi, \eta)=e^{i t\left(\xi^{3}+\eta^{3}\right)} \widehat{u_{0}}(\xi, \eta)
$$

where $S=-\partial_{x}^{3}-\partial_{y}^{3}$. Let us define $U_{S}^{p}=e^{\cdot S} U^{p}$ with norm $\|u\|_{U_{S}^{p}}=\left\|e^{-\cdot S} u\right\|_{U^{p}}$ and $V_{S}^{p}=e^{\cdot S} V^{p}$ with norm $\|v\|_{V_{S}^{p}}=\left\|e^{-\cdot S} v\right\|_{V^{p}}$.

Given the Littlewood-Paley multipliers by

$$
\widehat{P_{1} u}=\chi(2|\zeta|) \widehat{u}
$$

and

$$
\widehat{P_{N} u}=\psi_{N}(|\zeta|) \widehat{u} \quad \text { for } \quad N \geqslant 2
$$

where $\psi(x)=\chi(x)-\chi(2 x)$ and $\psi_{N}=\psi\left(N^{-1} \cdot\right)$, the smooth projections according to the dispersive relationship are defined by

$$
\begin{gathered}
\mathscr{F}\left(Q_{M} u\right)(\xi, \eta, \tau)=\psi_{M}(\tau) \tilde{u}(\xi, \eta, \tau), \\
\mathscr{F}\left(Q_{M}^{S} u\right)(\xi, \eta, \tau)=\psi_{M}\left(\tau-\xi^{3}-\eta^{3}\right) \tilde{u}(\xi, \eta, \tau),
\end{gathered}
$$

as well as $Q_{\geqslant M}^{S}=\sum_{N \geqslant M} Q_{N}^{S}$ and $Q_{<M}^{S}=I-Q_{\geqslant M}^{S}$. Note that $Q_{M}^{S}=e^{\cdot S} Q_{M} e^{-\cdot S}$.

Let's recall some useful results in $U^{p}$ and $V^{p}$.

Proposition 2.1. Let $1<p<q<\infty$ and $\frac{1}{p}+\frac{1}{p^{\prime}}=1$. We have

(i) $U^{p}, V^{p}, V_{r c}^{p}, V_{-}^{p}$ and $V_{-, r c}^{p}$ are Banach spaces,

(ii) $U^{p} \subset V_{-, r c}^{p} \subset U^{q}$,

(iii) $\|u\|_{U^{p}}=\sup _{\|v\|_{V p^{\prime}}=1}\left|\int\left\langle u^{\prime}(t), v(t)\right\rangle d t\right|$ if $u \in V_{-}^{1}\left(\subset U^{p}\right)$ is absolutely continuous on compact interval.

Lemma 2.2. We have

$$
\begin{gathered}
\left\|Q_{\geqslant M}^{S} u\right\|_{L^{2}\left(\mathbb{R}^{3}\right)} \lesssim M^{-\frac{1}{2}}\|u\|_{V_{S}^{2}} . \\
\left\|Q_{\geqslant M}^{S} u\right\|_{U_{S}^{p}} \lesssim\|u\|_{U_{S}^{p}}, \quad\left\|Q_{<M}^{S} u\right\|_{U_{S}^{p}} \lesssim\|u\|_{U_{S}^{p}} . \\
\left\|Q_{\geqslant M}^{S} u\right\|_{V_{S}^{p}} \lesssim\|u\|_{V_{S}^{p}}, \quad\left\|Q_{<M}^{S} u\right\|_{V_{S}^{p}} \lesssim\|u\|_{V_{S}^{p}} .
\end{gathered}
$$

Similarly to Lemma 2.3 in [7] and Lemma 5.3 in [28, the extension principle also holds true for $U_{S}^{p}$ spaces. 
Proposition 2.3. Let $T_{0}: L^{2} \times \cdots \times L^{2} \rightarrow L_{\text {loc }}^{1}\left(\mathbb{R}^{2} ; \mathbb{C}\right)$ be a n-linear operator. Assume that for some $1<p, q<\infty$

$$
\left\|T_{0}\left(e^{\cdot S} \phi_{1}, \cdots, e^{\cdot S} \phi_{n}\right)\right\|_{L_{t}^{p}\left(\mathbb{R} ; L_{x, y}^{q}\left(\mathbb{R}^{2}\right)\right)} \lesssim \prod_{j=1}^{n}\left\|\phi_{j}\right\|_{L^{2}} .
$$

Then, there exists $T: U_{S}^{p} \times \cdots \times U_{S}^{p} \rightarrow L_{t}^{p}\left(\mathbb{R} ; L_{x, y}^{q}\left(\mathbb{R}^{2}\right)\right)$ satisfying

$$
\left\|T\left(u_{1}, \cdots, u_{n}\right)\right\|_{L_{t}^{p}\left(\mathbb{R} ; L_{x, y}^{q}\left(\mathbb{R}^{2}\right)\right)} \lesssim \prod_{j=1}^{n}\left\|u_{j}\right\|_{U_{S}^{p}}
$$

such that $T\left(u_{1}, \cdots, u_{n}\right)(t)(x, y)=T_{0}\left(u_{1}(t), \cdots, u_{n}(t)\right)(x, y)$ a.e..

Next we present the Strichartz estimates and bilinear estimates (see Section 5 in [25]).

Lemma 2.4. Given $N_{1} \geqslant N_{2}$. Assume that $(q, r)$ satisfy $\frac{3}{q}+\frac{2}{r}=1$ and $q>3$. Let $I_{x,-}^{s}$ be the bilinear operator with symbol $\left|\xi_{1}-\xi_{2}\right|^{s}$, i.e.

$$
\mathscr{F}_{x, y}\left(I_{x,-}^{s}\left(f_{1}, f_{2}\right)\right)(\xi, \eta)=\int_{\zeta=\zeta_{1}+\zeta_{2}}\left|\xi_{1}-\xi_{2}\right|^{s} \prod_{j=1}^{2} \widehat{f}_{j}\left(\xi_{j}, \eta_{j}\right) .
$$

Then, we have

$$
\begin{gathered}
\left\|\chi\left(\frac{t}{T}\right) u\right\|_{L^{2}\left(\mathbb{R}^{3}\right)} \lesssim T^{\frac{1}{2}}\|u\|_{V_{S}^{2}}, \\
\|u\|_{L_{t}^{q} L_{x y}^{r}} \lesssim\|u\|_{U_{S}^{q}}, \\
\left\|I_{x}^{\frac{1}{8}} I_{y}^{\frac{1}{8}} e^{t S} u_{0}\right\|_{L^{4}\left(\mathbb{R}^{3}\right)} \lesssim\left\|u_{0}\right\|_{L^{2}\left(\mathbb{R}^{2}\right)}, \\
\left\|I_{x}^{\frac{1}{2}} I_{x,-}^{\frac{1}{2}}\left(P_{N_{1}} e^{t S} u_{0}, P_{N_{2}} e^{t S} v_{0}\right)\right\|_{L^{2}\left(\mathbb{R}^{3}\right)} \lesssim N_{2}^{\frac{1}{2}}\left\|P_{N_{1}} u_{0}\right\|_{L^{2}\left(\mathbb{R}^{2}\right)}\left\|P_{N_{2}} v_{0}\right\|_{L^{2}\left(\mathbb{R}^{2}\right)},
\end{gathered}
$$

Moreover, (2.7) is equally valid with $x$ replaced by $y$.

Lemma 2.5. Let $0 \leqslant \epsilon<\frac{1}{2}$ and $0 \leqslant \theta \leqslant 1$. Then,

$$
\left\|\tilde{D}^{\frac{\theta \epsilon}{2}} e^{t S} u_{0}\right\|_{L_{t}^{q} L_{x y}^{r}} \lesssim\left\|u_{0}\right\|_{L_{x y}^{2}},
$$

where $\widehat{\tilde{D}^{s} u}=|\xi+\eta|^{s} \hat{u}(\xi, \eta), q=\frac{6}{\theta(2+\epsilon)}$ and $r=\frac{2}{1-\theta}$. 
Proof. Set $\tilde{I}_{t}(x, y)=\int_{\mathbb{R}^{2}}|\xi+\eta|^{\epsilon+i \beta} e^{i\left[t\left(\xi^{3}+\eta^{3}\right)+(x \xi+y \eta)\right]} d \xi d \eta$. Let $\xi^{\prime}=4^{-\frac{1}{3}}(\xi+\eta)$ and $\eta^{\prime}=\sqrt{3} 4^{-\frac{1}{3}}(\xi-\eta)$, then we get

$$
\tilde{I}_{t}(x, y)=c I_{t}\left(2^{-\frac{1}{3}}(x+y), 2^{-\frac{1}{3}} 3^{-\frac{1}{2}}(x-y)\right),
$$

where $I_{t}(x, y)$ is defined as in Lemma 2.1 of [19. This implies

$$
\tilde{I}_{t}(x, y) \lesssim|t|^{-\frac{2+\epsilon}{3}}
$$

Hence, (2.8) is a direct consequence of interpolation theorem and standard Stein-Thomas argument.

We can avail ourselves of the technique in [22] to decompose the time cut-off into low- and high-frequency parts.

For any $\delta>0$, we write $1_{\delta}$ the characteristic function on $[0, \delta]$ and

$$
1_{\delta}=1_{\delta, \kappa}^{\text {low }}+1_{\delta, \kappa}^{\text {high }}, \widehat{1_{\delta, \kappa}^{\text {low }}}(\tau)=\chi(\tau / \kappa) \widehat{1_{\delta}}(\tau)
$$

for some $\kappa>0$.

Lemma 2.6. For any $\kappa, \delta>0$, it holds

$$
\begin{gathered}
\left\|1_{\delta, \kappa}^{h i g h}\right\|_{L^{\frac{3}{2}(\mathbb{R})}} \lesssim \delta^{\frac{1}{3}} \kappa^{-\frac{1}{3}}, \\
\left\|1_{\delta, \kappa}^{h i g h}\right\|_{L^{\infty}} \lesssim 1 \\
\left\|1_{\delta, \kappa}^{\text {low }}\right\|_{L^{\infty}} \lesssim 1 .
\end{gathered}
$$

\section{Global well-posedness}

We introduce $I$-method. Given $m: \mathbb{R}^{2 k} \rightarrow \mathbb{C}, m$ is said to be symmetric if

$$
m\left(\zeta_{1}, \cdots, \zeta_{k}\right)=m\left(\sigma\left(\zeta_{1}, \cdots, \zeta_{k}\right)\right)
$$

for all $\sigma \in S_{k}$, where $S_{k}$ is the permutation group for $k$ elements. The symbol $m$ can be symmetrized as following

$$
[m]_{s y m}\left(\zeta_{1}, \cdots, \zeta_{k}\right)=\frac{1}{k !} \sum_{\sigma \in S_{k}} m\left(\sigma\left(\zeta_{1}, \cdots, \zeta_{k}\right)\right)
$$

We define a $k$-linear functional acting on $k$ functions $u_{1}, \cdots, u_{k}$ for each $m$

$$
\Lambda_{k}\left(m ; u_{1}, \cdots, u_{k}\right)=\int_{\zeta_{1}+\cdots+\zeta_{k}=0} m\left(\zeta_{1}, \cdots, \zeta_{k}\right) \widehat{u}_{1}\left(\zeta_{k}\right) \cdots \widehat{u}_{k}\left(\zeta_{k}\right)
$$


Usually we write $\Lambda_{k}(m)$ instead of $\Lambda_{k}(m ; u, \cdots, u)$ for convenience. Note that $\Lambda_{k}(m)=$ $\Lambda_{k}\left([m]_{\text {sym }}\right)$ from symmetries.

Given $s<1, N \gg 1$ and a smooth, radially symmetric, nonincreasing function $m(\zeta)$ satisfying

$$
m(\zeta)=\left\{\begin{array}{ll}
1 & |\zeta| \leqslant N \\
\left(\frac{|\zeta|}{N}\right)^{s-1} & |\zeta| \geqslant 2 N
\end{array},\right.
$$

we define the Fourier multiplier operator

$$
\widehat{I f}(\zeta)=m(\zeta) \widehat{f}(\zeta)
$$

Let $\lambda>0$ and $N^{\prime}=\frac{N}{\lambda}$, we define

$$
m^{\prime}(\zeta)= \begin{cases}1 & |\zeta| \leqslant N^{\prime} \\ \left(\frac{|\zeta|}{N^{\prime}}\right)^{s-1} & |\zeta| \geqslant 2 N^{\prime}\end{cases}
$$

and rescaled operator

$$
\widehat{I^{\prime} f}(\zeta)=m^{\prime}(\zeta) \widehat{f}(\zeta)
$$

Notice that

$$
\left|\int \partial_{x} u \partial_{y} u d x d y\right| \leqslant \frac{1}{2} \int|\nabla u|^{2} d x d y
$$

it's easy to prove $\|u\|_{H^{s}}$ and $E(I u)(t)$ are comparable as Proposition 3.2 in [25].

Proposition 3.1. Let $\frac{5}{7}<s<1$, then

$$
\begin{gathered}
|E(I u)(t)| \lesssim N^{2(1-s)}\|u(t)\|_{\dot{H}^{s}\left(\mathbb{R}^{2}\right)}^{2}+\|u(t)\|_{L^{3}\left(\mathbb{R}^{2}\right)}^{3} \\
\|u(t)\|_{H^{s}\left(\mathbb{R}^{2}\right)}^{2} \lesssim|E(I u)(t)|+\left\|u_{0}\right\|_{L^{2}\left(\mathbb{R}^{2}\right)}^{2}+\left\|u_{0}\right\|_{L^{2}\left(\mathbb{R}^{2}\right)}^{4} .
\end{gathered}
$$

We start from local existence theroem. The work space is denoted by $Y^{s}$ which can be defined via the norm

$$
\|u\|_{Y^{s}}=\left(\sum_{N} N^{2 s}\left\|P_{N} u\right\|_{U_{S}^{2}}^{2}\right)^{\frac{1}{2}} .
$$

Obviously, $Y^{s} \subset L^{\infty} H^{s}$ holds true.

Lemma 3.2. Let $N_{1}, N_{2}$ be dyadic numbers and $N_{2} \lesssim N_{1}$. Assume that $\left|\zeta_{k}\right| \sim N_{k}$ $(k=1,2)$ and $\left|\zeta_{1}+\zeta_{2}\right| \sim N_{3}$. Denote $\Omega_{1}=\left\{\lambda_{1}+\lambda_{2}=\lambda,|\eta| \leqslant|\xi|,|\xi| \lesssim\left|\xi_{1}-\xi_{2}\right|\right\}$ and $\Omega_{2}=\left\{\lambda_{1}+\lambda_{2}=\lambda,|\eta| \leqslant|\xi|,|\xi| \sim\left|\xi_{1}\right| \sim\left|\xi_{2}\right| \lesssim\left|\eta_{1}\right| \sim\left|\eta_{2}\right|\right\}$. We have

(i) if $N_{1} \sim N_{3} \gg N_{2}$, then

$$
\left\|\int_{\Omega_{1}}(\xi+\eta) \frac{m\left(\zeta_{1}+\zeta_{2}\right)}{m\left(\zeta_{1}\right) m\left(\zeta_{2}\right)} \tilde{u}_{N_{1}}\left(\lambda_{1}\right) \tilde{v}_{N_{2}}\left(\lambda_{2}\right) d \lambda_{1}\right\|_{L_{\lambda}^{2}\left(\mathbb{R}^{3}\right)} \lesssim N_{2}^{\frac{3}{2}-s}\left\|u_{N_{1}}\right\|_{U_{S}^{2}}\left\|v_{N_{2}}\right\|_{U_{S}^{2}},
$$


(ii) if $N_{1} \sim N_{2} \gtrsim N_{3}$, then

$$
\left\|\int_{\Omega_{k}}(\xi+\eta) \frac{m\left(\zeta_{1}+\zeta_{2}\right)}{m\left(\zeta_{1}\right) m\left(\zeta_{2}\right)} \tilde{u}_{N_{1}}\left(\lambda_{1}\right) \tilde{v}_{N_{2}}\left(\lambda_{2}\right) d \lambda_{1}\right\|_{L_{\lambda}^{2}\left(\mathbb{R}^{3}\right)} \lesssim N_{1}^{\frac{1}{2}} C\left(N_{1}, N_{3}\right)\left\|u_{N_{1}}\right\|_{U_{S}^{2}}\left\|v_{N_{2}}\right\|_{U_{S}^{2}}
$$

for $k=1,2$, where

$$
C\left(N_{1}, N_{3}\right)=\left\{\begin{array}{ll}
1 & N \gg N_{1} \sim N_{2} \gg N_{3} \\
\left(\frac{N_{1}}{N}\right)^{2-2 s} & N_{1} \sim N_{2} \gtrsim N \gg N_{3} \\
N^{s-1} N_{1}{ }^{2-2 s} N_{3}{ }^{s-1} & N_{1} \sim N_{2} \gg N_{3} \gtrsim N \\
N_{1}{ }^{1-s} & N_{1} \sim N_{2} \sim N_{3}
\end{array} .\right.
$$

Proof. It's suffices to show the estimates for free solutions by Proposition 2.3. To this end, we can prove it along the same line as the proof of Lemma 5.3 in [25].

If $N_{1} \sim N_{3} \gg N_{2}$, it is obvious that

$$
\left|\frac{m\left(\zeta_{1}+\zeta_{2}\right)}{m\left(\zeta_{1}\right) m\left(\zeta_{2}\right)}\right| \lesssim 1 \vee\left(\frac{N_{2}}{N}\right)^{1-s} \lesssim N_{2}^{1-s}
$$

Besides, we have $|\xi+\eta| \lesssim|\xi| \lesssim|\xi|^{\frac{1}{2}}\left|\xi_{1}-\xi_{2}\right|^{\frac{1}{2}}$ on $\Omega_{1}$. Therefore, (2.7) gives

$$
\begin{aligned}
& \left.\| \int_{\Omega_{1}}(\xi+\eta) \frac{m\left(\zeta_{1}+\zeta_{2}\right)}{m\left(\zeta_{1}\right) m\left(\zeta_{2}\right)} \mathscr{F}\left(e^{t S} u_{0, N_{1}}\right)\left(\lambda_{1}\right)\right) \mathscr{F}\left(e^{t S} v_{0, N_{2}}\right)\left(\lambda_{2}\right) d \lambda_{1} \|_{L_{\lambda}^{2}\left(\mathbb{R}^{3}\right)} \\
\lesssim & \left.N_{2}^{1-s} \| \int_{\Omega_{1}}|\xi|^{\frac{1}{2}}\left|\xi_{1}-\xi_{2}\right|^{\frac{1}{2}} \mathscr{F}\left(e^{t S} u_{0, N_{1}}\right)\left(\lambda_{1}\right)\right) \mathscr{F}\left(e^{t S} v_{0, N_{2}}\right)\left(\lambda_{2}\right) d \lambda_{1} \|_{L_{\lambda}^{2}\left(\mathbb{R}^{3}\right)} \\
\lesssim & N_{2}^{1-s}\left\|I_{x}^{\frac{1}{2}} I_{x,-}^{\frac{1}{2}}\left(e^{t S} u_{0, N_{1}}, e^{t S} v_{0, N_{2}}\right)\right\|_{L_{\lambda}^{2}\left(\mathbb{R}^{3}\right)} \\
\lesssim & N_{2}^{\frac{3}{2}-s}\left\|u_{0, N_{1}}\right\|_{L_{x, y}^{2}}\left\|v_{0, N_{2}}\right\|_{L_{x, y}^{2}} .
\end{aligned}
$$

If $N_{1} \sim N_{2} \gtrsim N_{3}$, we can bound $\frac{m\left(\zeta_{1}+\zeta_{2}\right)}{m\left(\zeta_{1}\right) m\left(\zeta_{2}\right)}$ by $C\left(N_{1}, N_{3}\right)$. Also, on hyperplane $\Omega_{2}$, $|\xi+\eta| \lesssim|\xi| \lesssim N_{2}^{\frac{1}{2}}\left|\xi_{1}\right|^{\frac{1}{8}}\left|\eta_{1}\right|^{\frac{1}{8}}\left|\xi_{2}\right|^{\frac{1}{8}}\left|\eta_{2}\right|^{\frac{1}{8}}$. From Hörder inequalities and (2.6) , we get

$$
\begin{aligned}
& \left\|\int_{\Omega_{2}}(\xi+\eta) \frac{m\left(\zeta_{1}+\zeta_{2}\right)}{m\left(\zeta_{1}\right) m\left(\zeta_{2}\right)} \mathscr{F}\left(e^{t S} u_{0, N_{1}}\right)\left(\lambda_{1}\right) \mathscr{F}\left(e^{t S} v_{0, N_{2}}\right)\left(\lambda_{2}\right) d \lambda_{1}\right\|_{L_{\lambda}^{2}\left(\mathbb{R}^{3}\right)} \\
\lesssim & N_{1}^{\frac{1}{2}} C\left(N_{1}, N_{3}\right)\left\|\int_{\Omega_{2}} \mathscr{F}\left(I_{x}^{\frac{1}{8}} I_{y}^{\frac{1}{8}} e^{t S} u_{0, N_{1}}\right)\left(\lambda_{1}\right) \mathscr{F}\left(I_{x}^{\frac{1}{8}} I_{y}^{\frac{1}{8}} e^{t S} v_{0, N_{2}}\right)\left(\lambda_{2}\right) d \lambda_{1}\right\|_{L_{\lambda}^{2}\left(\mathbb{R}^{3}\right)} \\
\lesssim & N_{1}^{\frac{1}{2}} C\left(N_{1}, N_{3}\right)\left\|I_{x}^{\frac{1}{8}} I_{y}^{\frac{1}{8}} e^{t S} u_{0, N_{1}}\right\|_{L^{4}\left(\mathbb{R}^{3}\right)}\left\|I_{x}^{\frac{1}{8}} I_{y}^{\frac{1}{8}} e^{t S} v_{0, N_{2}}\right\|_{L^{4}\left(\mathbb{R}^{3}\right)} \\
\lesssim & N_{1}^{\frac{1}{2}} C\left(N_{1}, N_{3}\right)\left\|u_{0, N_{1}}\right\|_{L_{x, y}^{2}}\left\|v_{0, N_{2}}\right\|_{L_{x, y}^{2}} .
\end{aligned}
$$

Additionally, the estimate holds true on $\Omega_{1}$ in a similar way. Hence, the proof is completed. 
Lemma 3.3. Let $N_{1}, N_{2}, N_{3}$ be dyadic numbers and $C\left(N_{1}, N_{3}\right)$ be given as in Lemma 3.2. Then, for all $0<\delta \leqslant 1$ it holds

(i) if $N_{1} \sim N_{3} \gg N_{2}$,

$$
\begin{aligned}
& \left|\int_{\mathbb{R}^{3}} \chi\left(\frac{t}{\delta}\right) I\left(u_{N_{1}} v_{N_{2}}\right)\left(\partial_{x}+\partial_{y}\right) w_{N_{3}} d x d y d t\right| \\
\lesssim & \delta^{\frac{1}{2}} N_{2}^{\frac{3}{2}-s}\left\|I u_{N_{1}}\right\|_{U_{S}^{2}}\left\|I v_{N_{2}}\right\|_{U_{S}^{2}}\left\|w_{N_{3}}\right\|_{V_{S}^{2}},
\end{aligned}
$$

(ii) if $N_{1} \sim N_{2} \gtrsim N_{3}$,

$$
\begin{aligned}
& \left|\int_{\mathbb{R}^{3}} \chi\left(\frac{t}{\delta}\right) I\left(u_{N_{1}} v_{N_{2}}\right)\left(\partial_{x}+\partial_{y}\right) w_{N_{3}} d x d y d t\right| \\
\lesssim & \delta^{\frac{1}{6}} N_{1}^{\frac{1}{2}} C\left(N_{1}, N_{3}\right)\left\|I u_{N_{1}}\right\|_{U_{S}^{2}}\left\|I v_{N_{2}}\right\|_{U_{S}^{2}}\left\|w_{N_{3}}\right\|_{V_{S}^{2}} .
\end{aligned}
$$

Proof. As the technique used in Proposition 5.4 of [25] is applicative here, we just give a sketch to avoid being lengthy and tedious.

Parseval formula shows that it suffices to control

$$
\int_{\sum_{j=1}^{3} \lambda_{j}=0}\left(\xi_{3}+\eta_{3}\right) \frac{m\left(\zeta_{1}+\zeta_{2}\right)}{m\left(\zeta_{1}\right) m\left(\zeta_{2}\right)} \tilde{u}_{N_{1}}\left(\lambda_{1}\right) \tilde{v}_{N_{2}}\left(\lambda_{2}\right) \mathscr{F}\left(\chi_{\delta} w_{N_{3}}\right)\left(\lambda_{3}\right)
$$

by $\left\|u_{N_{1}}\right\|_{U_{S}^{2}},\left\|v_{N_{2}}\right\|_{U_{S}^{2}}$ and $\left\|w_{N_{3}}\right\|_{V_{S}^{2}}$.

We assume that $\left|\eta_{3}\right| \leqslant\left|\xi_{3}\right|$ and denote the absolute value of the quatity above by $R$.

If $N_{1} \sim N_{3} \gg N_{2}$, then $\left|\xi_{3}\right| \lesssim\left|\xi_{1}-\xi_{2}\right|$. Applying Cauchy-Schwarz inequality, (3.1) and (2.4) gives

$$
\begin{aligned}
R & \lesssim\left\|\int_{\Omega_{1}}(\xi+\eta) \frac{m\left(\zeta_{1}+\zeta_{2}\right)}{m\left(\zeta_{1}\right) m\left(\zeta_{2}\right)} \tilde{u}_{N_{1}}\left(\lambda_{1}\right) \tilde{v}_{N_{2}}\left(\lambda_{2}\right) d \lambda_{1}\right\|_{L_{\lambda}^{2}\left(\mathbb{R}^{3}\right)}\left\|\chi\left(\frac{t}{\delta}\right) w_{N_{3}}\right\|_{L^{2}\left(\mathbb{R}^{3}\right)} \\
& \lesssim \delta^{\frac{1}{2}} N_{2}^{\frac{3}{2}-s}\left\|u_{N_{1}}\right\|_{U_{S}^{2}}\left\|v_{N_{2}}\right\|_{U_{S}^{2}}\left\|w_{N_{3}}\right\|_{V_{S}^{2}} .
\end{aligned}
$$

So as to prove (3.4), we need to split the domain of the integration into five regions $R=R_{1}+R_{2}+R_{3}+R_{4}+R_{5}$. One can assume $\left|\eta_{1}\right| \geqslant\left|\eta_{2}\right|$ by symmetry.

Region 1. $\left|\xi_{3}\right| \lesssim\left|\xi_{1}-\xi_{2}\right|$

Using (3.2), we have

$$
\begin{aligned}
R_{1} & \lesssim\left\|\int_{\Omega_{1}}(\xi+\eta) \frac{m\left(\zeta_{1}+\zeta_{2}\right)}{m\left(\zeta_{1}\right) m\left(\zeta_{2}\right)} \tilde{u}_{N_{1}}\left(\lambda_{1}\right) \tilde{v}_{N_{2}}\left(\lambda_{2}\right) d \lambda_{1}\right\|_{L_{\lambda}^{2}\left(\mathbb{R}^{3}\right)}\left\|\chi\left(\frac{t}{\delta}\right) w_{N_{3}}\right\|_{L^{2}\left(\mathbb{R}^{3}\right)} \\
& \lesssim \delta^{\frac{1}{2}} N_{1}^{\frac{1}{2}} C\left(N_{1}, N_{3}\right)\left\|u_{N_{1}}\right\|_{U_{S}^{2}}\left\|v_{N_{2}}\right\|_{U_{S}^{2}}\left\|w_{N_{3}}\right\|_{V_{S}^{2}} .
\end{aligned}
$$

Region 2. $\left|\xi_{3}\right| \gg\left|\xi_{1}-\xi_{2}\right|$ and $\left|\eta_{1}\right| \gg\left|\xi_{3}\right|$ 
This condition gives

$$
\left|\eta_{1}\right| \sim\left|\eta_{2}\right| \gg\left|\xi_{3}\right| \sim\left|\xi_{1}\right| \sim\left|\xi_{2}\right| .
$$

Applying Cauchy-Schwarz inequality, (3.2) and (2.4), we obtain

$$
\begin{aligned}
R_{2} & \lesssim\left\|\int_{\Omega_{2}}(\xi+\eta) \frac{m\left(\zeta_{1}+\zeta_{2}\right)}{m\left(\zeta_{1}\right) m\left(\zeta_{2}\right)} \tilde{u}_{N_{1}}\left(\lambda_{1}\right) \tilde{v}_{N_{2}}\left(\lambda_{2}\right) d \lambda_{1}\right\|_{L^{2}\left(\mathbb{R}^{3}\right)}\left\|\chi\left(\frac{t}{\delta}\right) w_{N_{3}}\right\|_{L^{2}\left(\mathbb{R}^{3}\right)} \\
& \lesssim \delta^{\frac{1}{2}} N_{1}^{\frac{1}{2}} C\left(N_{1}, N_{3}\right)\left\|u_{N_{1}}\right\|_{U_{S}^{2}}\left\|v_{N_{2}}\right\|_{U_{S}^{2}}\left\|w_{N_{3}}\right\|_{V_{S}^{2}} .
\end{aligned}
$$

Region 3. $\left|\xi_{3}\right| \gg\left|\xi_{1}-\xi_{2}\right|$ and $\left|\eta_{1}\right| \sim\left|\xi_{3}\right| \sim\left|\eta_{2}\right|$

We get the bound of $R_{3}$ like Region 2 .

Region 4. $\left|\xi_{3}\right| \gg\left|\xi_{1}-\xi_{2}\right|$ and $\left|\eta_{1}\right| \sim\left|\xi_{3}\right| \gg\left|\eta_{2}\right|$

We decompose $I d=Q_{<M}^{S}+Q_{\geqslant M}^{S}$ and divide the integral $R_{4}$ into eight pieces.

Case A. $Q_{j}^{S}=Q_{<M}^{S}$ for $j=1,2,3$

We go a step further to decompose time into low- and high-frequency parts.

Case A(1). All of these three are low-frequence

The integral is vanished.

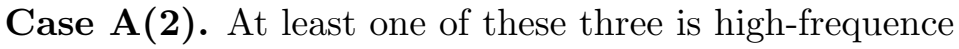

We estimate when the first one is high-frequence

$$
\int_{*}\left(\xi_{3}+\eta_{3}\right) \frac{m\left(\zeta_{1}+\zeta_{2}\right)}{m\left(\zeta_{1}\right) m\left(\zeta_{2}\right)} \mathscr{F}\left(1_{\delta, \kappa}^{h i g h} Q_{<M}^{S} u_{N_{1}}\right) \mathscr{F}\left(1_{v} Q_{<M}^{S} v_{N_{2}}\right) \mathscr{F}\left(1_{w} Q_{<M}^{S} w_{N_{3}}\right),
$$

where $1_{v}, 1_{w} \in\left\{1_{\delta, \kappa}^{\text {high }}, 1_{\delta, \kappa}^{\text {low }}\right\}$.

Hölder inequalities, (2.5), Lemma 2.6 and (2.2) provide

$$
\begin{aligned}
& \left|\int_{*}\left(\xi_{3}+\eta_{3}\right) \frac{m\left(\zeta_{1}+\zeta_{2}\right)}{m\left(\zeta_{1}\right) m\left(\zeta_{2}\right)} \mathscr{F}\left(1_{\delta, \kappa}^{h i g h} Q_{<M}^{S} u_{N_{1}}\right) \mathscr{F}\left(1_{v} Q_{<M}^{S} v_{N_{2}}\right) \mathscr{F}\left(1_{w} Q_{<M}^{S} w_{N_{3}}\right)\right| \\
\lesssim & \left\|1_{\delta, \kappa}^{h i g h} Q_{<M}^{S} u_{N_{1}}^{*}\right\|_{L_{t}^{\frac{9}{7}} L_{x y}^{3}}\left\|1_{v} Q_{<M}^{S} v_{N_{2}}^{*}\right\|_{L_{t}^{9} L_{x y}^{3}}\left\|1_{w} Q_{<M}^{S} w_{N_{3}}^{*}\right\|_{L_{t}^{9} L_{x y}^{3}} \\
\lesssim & \left\|1_{\delta, L}^{h i g h}\right\|_{L_{t}^{\frac{3}{2}}}\left\|Q_{<M}^{S} u_{N_{1}}^{*}\right\|_{L_{t}^{9} L_{x y}^{3}}\left\|1_{v}\right\|_{L_{t}^{\infty}}\left\|Q_{<M}^{S} v_{N_{2}}^{*}\right\|_{L_{t}^{9} L_{x y}^{3}}\left\|1_{w}\right\|_{L_{t}^{\infty}}\left\|Q_{<M}^{S} w_{N_{3}}^{*}\right\|_{L_{t}^{9} L_{x y}^{3}} \\
\lesssim & \delta^{\frac{1}{3}} N_{3}^{-\frac{1}{3}} N_{1}^{-\frac{2}{3}}\left\|Q_{<M}^{S} u_{N_{1}}^{*}\right\|_{U_{S}^{9}}\left\|Q_{<M}^{S} v_{N_{2}}^{*}\right\|_{U_{S}^{9}}\left\|Q_{<M}^{S} w_{N_{3}}^{*}\right\|_{U_{S}^{9}} \\
\lesssim & \delta^{\frac{1}{3}} N_{3}^{-\frac{1}{3}} N_{1}^{-\frac{2}{3}}\left\|u_{N_{1}}^{*}\right\|_{U_{S}^{2}}\left\|v_{N_{2}}^{*}\right\|_{U_{S}^{2}}\left\|w_{N_{3}}^{*}\right\|_{V_{S}^{2}},
\end{aligned}
$$

where $u_{N_{1}}^{*}=\mathscr{F}^{-1} \frac{\widehat{u}\left(\zeta_{1}\right)}{m\left(\zeta_{1}\right)}, v_{N_{2}}^{*}=\mathscr{F}^{-1} \frac{\widehat{v}\left(\zeta_{2}\right)}{m\left(\zeta_{2}\right)}$ and $w_{N_{3}}^{*}=\mathscr{F}^{-1}\left(\xi_{3}+\eta_{3}\right) m\left(\zeta_{3}\right) \widehat{w}\left(\zeta_{3}\right)$.

From the definition of $U^{2}$ and $V^{2}$, there hold the facts

$$
\left\|u_{N_{1}}^{*}\right\|_{U_{S}^{2}} \lesssim \frac{\left\|u_{N_{1}}\right\|_{U_{S}^{2}}}{m\left(N_{1}\right)}, \quad\left\|v_{N_{2}}^{*}\right\|_{U_{S}^{2}} \lesssim \frac{\left\|v_{N_{2}}\right\|_{U_{S}^{2}}}{m\left(N_{2}\right)}
$$

and

$$
\left\|w_{N_{3}}^{*}\right\|_{V_{S}^{2}} \lesssim N_{3} m\left(N_{3}\right)\left\|w_{N_{3}}\right\|_{V_{S}^{2}}
$$


which of course help imply the desired up bound

$$
\delta^{\frac{1}{3}} N_{1}^{\frac{1}{2}} C\left(N_{1}, N_{3}\right)\left\|u_{N_{1}}\right\|_{U_{S}^{2}}\left\|v_{N_{2}}\right\|_{U_{S}^{2}}\left\|w_{N_{3}}\right\|_{V_{S}^{2}}
$$

Case B. $Q_{j}^{S}=Q_{\geqslant M}^{S}$ for some $j=1,2,3$

We take $Q_{3}^{S}=Q_{\geqslant M}^{S}$ for instance to give the estimate.

Using Hölder inequalities, (2.1), (2.5) and (2.2), we have

$$
\begin{aligned}
& \left|\int_{*}\left(\xi_{3}+\eta_{3}\right) \frac{m\left(\zeta_{1}+\zeta_{2}\right)}{m\left(\zeta_{1}\right) m\left(\zeta_{2}\right)} \mathscr{F}\left(\chi_{\delta} Q_{1}^{S} u_{N_{1}}\right) \mathscr{F}\left(\chi_{\delta} Q_{2}^{S} v_{N_{2}}\right) \mathscr{F}\left(\chi_{\delta} Q_{\geqslant M}^{S} w_{N_{3}}\right)\right| \\
& \lesssim\left\|\chi_{\delta} Q_{1}^{S} u_{N_{1}}^{*}\right\|_{L^{4}\left(\mathbb{R}^{3}\right)}\left\|\chi_{\delta} Q_{2}^{S} v_{N_{2}}^{*}\right\|_{L^{4}\left(\mathbb{R}^{3}\right)}\left\|Q_{\geqslant M}^{S} w_{N_{3}}^{*}\right\|_{L^{2}\left(\mathbb{R}^{3}\right)} \\
& \lesssim M^{-\frac{1}{2}}\left\|\chi_{\delta}\right\|_{L^{12}(\mathbb{R})}^{2}\left\|Q_{1}^{S} u_{N_{1}}^{*}\right\|_{L_{t}^{6} L_{x, y}^{4}}\left\|Q_{2}^{S} v_{N_{2}}^{*}\right\|_{L_{t}^{6} L_{x, y}^{4}}\left\|w_{N_{3}}^{*}\right\|_{V_{S}^{2}} \\
& \lesssim \delta^{\frac{1}{6}} N_{1}^{-1} N_{3}^{-\frac{1}{2}}\left\|Q_{1}^{S} u_{N_{1}}^{*}\right\|_{U_{S}^{6}}\left\|Q_{2}^{S} v_{N_{2}}^{*}\right\|_{U_{S}^{6}}\left\|w_{N_{3}}^{*}\right\|_{V_{S}^{2}} \\
& \lesssim \delta^{\frac{1}{6}} N_{1}^{\frac{1}{2}} C\left(N_{1}, N_{3}\right)\left\|u_{N_{1}}\right\|_{U_{S}^{2}}\left\|v_{N_{2}}\right\|_{U_{S}^{2}}\left\|w_{N_{3}}\right\|_{V_{S}^{2}} \text {. }
\end{aligned}
$$

Region 5. $\left|\xi_{3}\right| \gg\left|\xi_{1}-\xi_{2}\right|$ and $\left|\xi_{3}\right| \gg\left|\eta_{1}\right| \geqslant\left|\eta_{2}\right|$

This can be dealt with in the same way as Region 4 .

Next, we turn to the estimate of the nonlinear term.

Proposition 3.4. Let $\frac{5}{7}<s<1$ and $0<\delta \leqslant 1$. We have

$$
\left\|\int_{0}^{t} e^{\left(t-t^{\prime}\right) S} \chi\left(\frac{t}{\delta}\right)\left(\partial_{x}+\partial_{y}\right) I(u v)\left(t^{\prime}\right) d t^{\prime}\right\|_{Y^{1}} \lesssim \delta^{\frac{1}{6}}\|I u\|_{Y^{1}}\|I v\|_{Y^{1}}
$$

Proof. From symmetry and definition of $Y^{1}$ we need to consider the following two terms

$$
J_{1}=\sum_{N_{3}} N_{3}^{2}\left\|\sum_{N_{1} \gg N_{2}} \int_{0}^{t} e^{\left(t-t^{\prime}\right) S} \chi\left(\frac{t}{\delta}\right)\left(\partial_{x}+\partial_{y}\right) P_{N_{3}} I\left(u_{N_{1}} v_{N_{2}}\right)\left(t^{\prime}\right) d t^{\prime}\right\|_{U_{S}^{2}}^{2}
$$

and

$$
J_{2}=\sum_{N_{3}} N_{3}^{2}\left\|\sum_{N_{1} \sim N_{2}} \int_{0}^{t} e^{\left(t-t^{\prime}\right) S} \chi\left(\frac{t}{\delta}\right)\left(\partial_{x}+\partial_{y}\right) P_{N_{3}} I\left(u_{N_{1}} v_{N_{2}}\right)\left(t^{\prime}\right) d t^{\prime}\right\|_{U_{S}^{2}}^{2} .
$$

Combining Proposition 2.1 (iii) with (3.3), we obtain

$$
\begin{aligned}
J_{1} & \lesssim \sum_{N_{3}} N_{3}^{2}\left(\sum_{N_{1} \gg N_{2}} \sup _{\left\|w_{N_{3}}\right\|_{V_{S}^{2}} \leqslant 1}\left|\int_{\mathbb{R}^{3}} \chi\left(\frac{t}{\delta}\right) I\left(u_{N_{1}} v_{N_{2}}\right)\left(\partial_{x}+\partial_{y}\right) w_{N_{3}} d x d y d t\right|\right)^{2} \\
& \lesssim \delta \sum_{N_{3}} N_{3}^{2}\left(\sum_{N_{1} \gg N_{2}} N_{2}^{\frac{3}{2}-s}\left\|I u_{N_{1}}\right\|_{U_{S}^{2}}\left\|I v_{N_{2}}\right\|_{U_{S}^{2}}\right)^{2}
\end{aligned}
$$




$$
\begin{aligned}
& \lesssim \delta \sum_{N_{1}} N_{1}^{2}\left\|I u_{N_{1}}\right\|_{U_{S}^{2}}^{2} \sum_{N_{2}} N_{2}^{2}\left\|I v_{N_{2}}\right\|_{U_{S}^{2}}^{2} \sum_{N_{2}} N_{2}^{1-2 s} \\
& \lesssim \delta\|I u\|_{Y^{1}}^{2}\|I v\|_{Y^{1}}^{2} .
\end{aligned}
$$

The second term can be controlled via (3.4) and Minkowshi inequality,

$$
\begin{aligned}
J_{2} \lesssim & \sum_{N_{3}} N_{3}^{2}\left(\sum_{N_{1} \sim N_{2}} \sup _{\lesssim} \mid \int_{\mathbb{N}_{3}} \|_{V_{S}^{2}} \leqslant 1\right. \\
\lesssim & \delta^{\frac{1}{3}}\left(\sum_{N_{3} \sim N_{1}} N_{3}^{2}\left(N_{1}^{\frac{3}{2}-s}\left\|I u_{N_{1}}\right\|_{U_{S}^{2}}\left\|I v_{N_{1}}\right\|_{U_{S}^{2}}\right)^{2}\right. \\
& \left.+\sum_{N_{3} \ll N} N_{3}^{2}\left(\sum_{N \lesssim N_{1}} N_{1}^{\frac{1}{2}}\left(\frac{N_{1}}{N}\right)^{2-2 s}\left\|I u_{N_{1}}\right\|_{U_{S}^{2}}\left\|I v_{N_{1}}\right\|_{U_{S}^{2}}\right)^{2}\left(\partial_{x}+\partial_{y}\right) w_{N_{3}} d x d y d t \mid\right)^{2} \\
& \left.+\sum_{N \lesssim N_{3}} N_{3}^{2}\left(\sum_{N_{3} \ll N_{1}} N_{1}^{\frac{1}{2}} N^{s-1} N_{1}^{2-2 s} N_{3}^{s-1}\left\|I u_{N_{1}}\right\|_{U_{S}^{2}}\left\|I v_{N_{1}}\right\|_{U_{S}^{2}}\right)^{2}\right) \\
\lesssim & \delta^{\frac{1}{3}}\left(\sum_{N_{1}} N_{1}^{5-2 s}\left\|I u_{N_{1}}\right\|_{U_{S}^{2}}^{2}\left\|I v_{N_{1}}\right\|_{U_{S}^{2}}^{2}\right. \\
& +N^{4 s-2}\left(\sum_{N \lesssim N_{1}} N_{1}^{2} N_{1}^{\frac{1}{2}-2 s}\left\|I u_{N_{1}}\right\|_{U_{S}^{2}}\left\|I v_{N_{1}}\right\|_{U_{S}^{2}}\right)^{2} \\
& \left.+N^{2 s-2} \sum_{N_{3} \ll N_{1}} N_{3}^{2 s}\left(\sum_{N_{1}} N_{1}^{\frac{5}{2}-2 s}\left\|I u_{N_{1}}\right\|_{U_{S}^{2}}\left\|I v_{N_{1}}\right\|_{U_{S}^{2}}\right)^{2}\right) \\
\lesssim & \delta^{\frac{1}{3}}\left(\sum_{N_{1}} N_{1}^{4}\left\|I u_{N_{1}}\right\|_{U_{S}^{2}}^{2}\left\|I v_{N_{2}}\right\|_{U_{S}^{2}}^{2}+\left(\sum_{N_{1}} N_{1}^{2}\left\|I u_{N_{1}}\right\|_{U_{S}^{2}}\left\|I v_{N_{1}}\right\|_{U_{S}^{2}}\right)^{2}\right. \\
& \left.+\left(\sum_{N_{1}} N_{1}^{\frac{5}{2}-s}\left\|I u_{N_{1}}\right\|_{U_{S}^{2}}\left\|I v_{N_{1}}\right\|_{U_{S}^{2}}\right)^{2}\right) \\
\lesssim & \delta^{\frac{1}{3}}\|I u\|_{Y^{1}}^{2}\|I v\|_{Y^{1}}^{2} .
\end{aligned}
$$

Hence, we complete the proof.

Proposition 3.5. Let $\frac{5}{7}<s<1$. Assume $u_{0}$ satisfies $\left|E\left(I u_{0}\right)\right| \leqslant 1$. Then there is a constant $\delta=\delta\left(\left\|u_{0}\right\|_{L^{2}\left(\mathbb{R}^{2}\right)}\right)$ and a unique solution $u$ to (1.1) on $[0, \delta]$, such that

$$
\|I u\|_{Y^{1}} \lesssim 1
$$

where the implicit constant is independent of $\delta$.

Proof. Acting multiplier operator $I$ on both sides of (1.1), one can obtain

$$
\partial_{t} I u+\left(\partial_{x}^{3}+\partial_{y}^{3}\right) I u+\left(\partial_{x}+\partial_{y}\right) I\left(u^{2}\right)=0 .
$$


From Duhamel's principle, we get

$$
I u=\chi\left(\frac{t}{\delta}\right) e^{t S} I u_{0}-\int_{0}^{t} e^{\left(t-t^{\prime}\right) S} \chi\left(\frac{t}{\delta}\right)\left(\partial_{x}+\partial_{y}\right) I\left(u^{2}\right)\left(t^{\prime}\right) d t^{\prime} .
$$

Applying Proposition 3.4, one obtain

$$
\begin{aligned}
\|I u\|_{Y^{1}} & \leqslant\left\|\chi\left(\frac{t}{\delta}\right) e^{t S} I u_{0}\right\|_{Y^{1}}+\left\|\int_{0}^{t} e^{\left(t-t^{\prime}\right) S} \chi\left(\frac{t}{\delta}\right)\left(\partial_{x}+\partial_{y}\right) I\left(u^{2}\right)\left(t^{\prime}\right) d t^{\prime}\right\|_{Y^{1}} \\
& \leqslant\left\|I u_{0}\right\|_{H^{1}\left(\mathbb{R}^{2}\right)}+C \delta^{\frac{1}{6}}\|I u\|_{Y^{1}}^{2},
\end{aligned}
$$

and

$$
\|I u-I v\|_{Y^{1}} \leqslant C \delta^{\frac{1}{6}}\left(\|I u\|_{Y^{1}}+\|I v\|_{Y^{1}}\right)\|I u-I v\|_{Y^{1}} .
$$

Then, the contraction mapping principle ensure the existence of local solution. Moreover, a bootstrap argument yields $\|I u\|_{Y^{1}} \lesssim 1$.

According to equation (3.6) and integration by parts, we have

$$
\begin{aligned}
\frac{d E(I u)(t)}{d t} & =-\int_{\mathbb{R}^{2}}\left(\Delta I u-\partial_{x} \partial_{y} I u+(I u)^{2}\right) \partial_{t} I u d x d y \\
& =\int_{\mathbb{R}^{2}}\left(\left(\partial_{x}^{3}+\partial_{y}^{3}\right) I u+\left(\partial_{x}+\partial_{y}\right) I u^{2}\right)\left(\left(\Delta-\partial_{x} \partial_{y}\right) I u+(I u)^{2}\right) d x d y \\
& =\int_{\mathbb{R}^{2}}\left(\partial_{x}^{3}+\partial_{y}^{3}\right) I u\left((I u)^{2}-I u^{2}\right) d x d y+\int_{\mathbb{R}^{2}}\left(\partial_{x}+\partial_{y}\right) I u^{2}(I u)^{2} d x d y
\end{aligned}
$$

Integrating in time and applying the Parseval formula provide

$$
\begin{aligned}
E(I u)(\delta)-E(I u)(0) & =\int_{0}^{\delta} \int_{\sum_{j=1}^{3} \zeta_{j}=0}\left(\xi_{1}^{3}+\eta_{1}^{3}\right)\left(1-\frac{m\left(\zeta_{2}+\zeta_{3}\right)}{m\left(\zeta_{2}\right) m\left(\zeta_{3}\right)}\right) \prod_{j=1}^{3} \widehat{I u}\left(\zeta_{j}\right) \\
& +\int_{0}^{\delta} \int_{\sum_{j=1}^{4} \zeta_{j}=0}\left(\xi_{1}+\xi_{2}+\eta_{1}+\eta_{2}\right) \frac{m\left(\zeta_{1}+\zeta_{2}\right)}{m\left(\zeta_{1}\right) m\left(\zeta_{2}\right)} \prod_{j=1}^{4} \widehat{I u}\left(\zeta_{j}\right) .
\end{aligned}
$$

The following preliminaries are effective for controlling the growth of $E(I u)(t)$.

Lemma 3.6. Let $N_{1}, N_{2}, N_{3}$ be dyadic numbers. Then, for all $0<\delta \leqslant 1$ we have if $N_{1} \sim N_{2} \sim N_{3} \gtrsim N$,

$$
\begin{aligned}
& \quad\left|\int_{\sum_{j=1}^{3} \lambda_{j}=0}\left(\xi_{1}^{3}+\eta_{1}^{3}\right)\left(1-\frac{m\left(\zeta_{2}+\zeta_{3}\right)}{m\left(\zeta_{2}\right) m\left(\zeta_{3}\right)}\right) \prod_{j=1}^{3} \mathscr{F}\left(\chi_{\delta} u_{j}\right)\left(\lambda_{j}\right)\right| \\
& \delta^{\frac{1}{6}} N^{s-1} N_{1}^{\frac{7}{2}-s} \prod_{j=1}^{3}\left\|u_{j}\right\|_{U_{S}^{2}},
\end{aligned}
$$


if $N_{1} \sim N_{2} \gg N_{3}, N_{1} \gtrsim N$

$$
\begin{aligned}
& \left|\int_{\sum_{j=1}^{3} \lambda_{j}=0}\left(\xi_{1} \xi_{2} \xi_{3}+\eta_{1} \eta_{2} \eta_{3}\right) \prod_{j=1}^{3} \mathscr{F}\left(\chi_{\delta} u_{j}\right)\left(\lambda_{j}\right)\right| \\
& \lesssim \delta^{\frac{1}{2}} N_{1} N_{3}^{\frac{3}{2}} \prod_{j=1}^{3}\left\|u_{j}\right\|_{U_{S}^{2}},
\end{aligned}
$$

and

$$
\begin{aligned}
& \left|\int_{\sum_{j=1}^{3} \lambda_{j}=0} \frac{\sum_{j=1}^{3}\left(\xi_{j}^{3}+\eta_{j}^{3}\right) m^{2}\left(\zeta_{j}\right)}{m\left(\zeta_{1}\right) m\left(\zeta_{2}\right) m\left(\zeta_{3}\right)} \prod_{j=1}^{3} \mathscr{F}\left(\chi_{\delta} u_{j}\right)\left(\lambda_{j}\right)\right| \\
& \lesssim \delta^{\frac{1}{2}} N_{1} N_{3}^{\frac{3}{2}}\left(1 \vee\left(\frac{N_{3}}{N}\right)^{1-s}\right) \prod_{j=1}^{3}\left\|u_{j}\right\|_{U_{S}^{2}} .
\end{aligned}
$$

Proof. When $N_{1} \sim N_{2} \sim N_{3} \gtrsim N$,

$$
\left|1-\frac{m\left(\zeta_{2}+\zeta_{3}\right)}{m\left(\zeta_{2}\right) m\left(\zeta_{3}\right)}\right| \lesssim \frac{m\left(\zeta_{1}\right)}{m\left(\zeta_{2}\right) m\left(\zeta_{3}\right)} \lesssim\left(\frac{N_{1}}{N}\right)^{1-s}
$$

and

$$
\left|\xi_{1}^{3}+\eta_{1}^{3}\right| \lesssim\left|\xi_{1}+\eta_{1}\right| N_{1}^{2}
$$

Then one can get (3.8) as the second part of Lemma 3.3.

In order to prove (3.9), we assume $\left|\xi_{1} \xi_{2} \xi_{3}\right| \geqslant\left|\eta_{1} \eta_{2} \eta_{3}\right|$ by symmetry.

Case 1. $\left|\xi_{2}\right| \lesssim\left|\xi_{1}-\xi_{3}\right|$

From (2.7), we have

$$
\begin{aligned}
&\left|\int_{\sum_{j=1}^{3} \lambda_{j}=0}\left(\xi_{1} \xi_{2} \xi_{3}+\eta_{1} \eta_{2} \eta_{3}\right) \prod_{j=1}^{3} \mathscr{F}\left(\chi_{\delta} u_{j}\right)\left(\lambda_{j}\right)\right| \\
& \lesssim\left.N_{1} N_{3}\left|\int_{\sum_{j=1}^{3} \lambda_{j}=0}\right| \xi_{2}\right|^{\frac{1}{2}}\left|\xi_{1}-\xi_{3}\right|^{\frac{1}{2}} \prod_{j=1}^{3} \mathscr{F}\left(\chi_{\delta} u_{j}\right)\left(\lambda_{j}\right) \mid \\
& \lesssim N_{1} N_{3}\left\|\int\left|\xi_{1}+\xi_{3}\right|^{\frac{1}{2}}\left|\xi_{1}-\xi_{3}\right|^{\frac{1}{2}} \tilde{u}_{1}\left(\lambda_{1}\right) \tilde{u}_{3}\left(\lambda_{3}\right)\right\|_{L^{2}\left(\mathbb{R}^{3}\right)}\left\|\chi\left(\frac{t}{\delta}\right) u_{2}\right\|_{L^{2}\left(\mathbb{R}^{3}\right)} \\
& \lesssim \delta^{\frac{1}{2}} N_{1} N_{3}^{\frac{3}{2}} \prod_{j=1}^{3}\left\|u_{j}\right\|_{U_{S}^{2}} .
\end{aligned}
$$

Case 2. $\left|\xi_{2}\right| \gg\left|\xi_{1}-\xi_{3}\right|$

In this case, $\left|\xi_{2}\right| \sim\left|\xi_{1}\right| \sim\left|\xi_{3}\right| \lesssim N_{3} \ll N_{1} \sim N_{2}$. Hence,

$$
\left|\xi_{1} \xi_{2} \xi_{3}\right| \lesssim N_{3}^{\frac{5}{2}}\left|\xi_{1}\right|^{\frac{1}{8}}\left|\eta_{1}\right|^{\frac{1}{8}}\left|\xi_{2}\right|^{\frac{1}{8}}\left|\eta_{2}\right|^{\frac{1}{8}}
$$


From (2.6), we have

$$
\begin{aligned}
& \left|\int_{\sum_{j=1}^{3} \lambda_{j}=0}\left(\xi_{1} \xi_{2} \xi_{3}+\eta_{1} \eta_{2} \eta_{3}\right) \prod_{j=1}^{3} \mathscr{F}\left(\chi_{\delta} u_{j}\right)\left(\lambda_{j}\right)\right| \\
\lesssim & \left|\int_{\sum_{j=1}^{3} \lambda_{j}=0}\right| \xi_{1} \xi_{2} \xi_{3}\left|\prod_{j=1}^{3} \mathscr{F}\left(\chi_{\delta} u_{j}\right)\left(\lambda_{j}\right)\right| \\
\lesssim & \left.N_{3}^{\frac{5}{2}}\left|\int_{\sum_{j=1}^{3} \lambda_{j}=0}\right| \xi_{1}\right|^{\frac{1}{8}}\left|\eta_{1}\right|^{\frac{1}{8}}\left|\xi_{2}\right|^{\frac{1}{8}}\left|\eta_{2}\right|^{\frac{1}{8}} \prod_{j=1}^{3} \mathscr{F}\left(\chi_{\delta} u_{j}\right)\left(\lambda_{j}\right) \mid \\
\lesssim & N_{3}^{\frac{5}{2}}\left\|I_{x}^{\frac{1}{8}} I_{y}^{\frac{1}{8}} u_{1}\right\|_{L^{4}\left(\mathbb{R}^{3}\right)}\left\|I_{x}^{\frac{1}{8}} I_{y}^{\frac{1}{8}} u_{2}\right\|_{L^{4}\left(\mathbb{R}^{3}\right)}\left\|\chi\left(\frac{t}{\delta}\right) u_{3}\right\|_{L^{2}\left(\mathbb{R}^{3}\right)} \\
\lesssim & \delta^{\frac{1}{2}} N_{1} N_{3}^{\frac{3}{2}} \prod_{j=1}^{3}\left\|u_{j}\right\|_{U_{S}^{2}} .
\end{aligned}
$$

It's easy to know that $N_{1} \sim N_{2} \gg N_{3}$ implies $\left|\xi_{1}\right| \sim\left|\xi_{2}\right| \sim N_{1}$ or $\left|\eta_{1}\right| \sim\left|\eta_{2}\right| \sim N_{1}$. Without loss of generality one can $\left|\xi_{1}\right| \sim\left|\xi_{2}\right| \sim N_{1}$, then mean-value theorem gives the bound of the symbol

$$
\sum_{j=1}^{3}\left(\xi_{j}^{3}+\eta_{j}^{3}\right) m^{2}\left(\zeta_{j}\right) \lesssim N_{1}^{2} \max \left\{\left|\xi_{3}\right|,\left|\eta_{3}\right|\right\} m^{2}\left(\zeta_{1}\right) .
$$

Hence, one can show (3.10) in a similar way as above.

We complete the proof of this lemma.

Proposition 3.7. Let $\frac{5}{7}<s<1$. We have

$$
\left|\int_{0}^{\delta} \Lambda_{3}\left(\left(\xi_{1}^{3}+\eta_{1}^{3}\right)\left(1-\frac{m\left(\zeta_{2}+\zeta_{3}\right)}{m\left(\zeta_{2}\right) m\left(\zeta_{3}\right)}\right) ; I u\right)\right| \lesssim \delta^{\frac{1}{6}} N^{-\frac{1}{2}+}\|I u\|_{Y^{1}}^{3} .
$$

Proof. The fact

$$
\sum_{N_{1}} N_{1}^{0-} N_{1}\left\|P_{N_{1}} I u\right\|_{U_{S}^{2}} \leqslant\left(\sum_{N_{1}} N_{1}^{0-}\right)^{\frac{1}{2}}\|I u\|_{Y^{1}}
$$

indicates that it's suffices to prove

$$
\begin{aligned}
& \left|\int_{0}^{\delta} \int_{\sum_{j=1}^{3} \zeta_{j}=0}\left[\left(\xi_{1}^{3}+\eta_{1}^{3}\right)\left(1-\frac{m\left(\zeta_{2}+\zeta_{3}\right)}{m\left(\zeta_{2}\right) m\left(\zeta_{3}\right)}\right)\right]_{s y m} \prod_{j=1}^{3} \widehat{u_{j}}\left(\zeta_{j}\right)\right| \\
& \lesssim \delta^{\frac{1}{6}} N^{-\frac{1}{2}+}\left(N_{1} N_{2} N_{3}\right)^{1-} \prod_{j=1}^{3}\left\|u_{j}\right\|_{U_{S}^{2}}
\end{aligned}
$$

for any function $u_{j}(j=1,2,3)$ with frequencies supported on $\left|\zeta_{j}\right| \sim N_{j}$. 
We denote $L_{1}$ the left hand of (13.12) and $M\left(\zeta_{1}, \zeta_{2}, \zeta_{3}\right)=\left[\left(\xi_{1}^{3}+\eta_{1}^{3}\right)\left(1-\frac{m\left(\zeta_{2}+\zeta_{3}\right)}{m\left(\zeta_{2}\right) m\left(\zeta_{3}\right)}\right)\right]_{s y m}$. Furthermore, one can assume $N_{1} \sim N_{2} \gtrsim N_{3}$ and $N_{1} \gtrsim N$.

Case 1. $N_{1} \sim N_{2} \sim N_{3} \gtrsim N$

We obtain by (3.8)

$$
\begin{aligned}
L_{1} & \lesssim \delta^{\frac{1}{6}} N^{s-1} N_{1}^{\frac{7}{2}-s} \prod_{j=1}^{3}\left\|u_{j}\right\|_{U_{S}^{2}} \\
& \lesssim \delta^{\frac{1}{6}} N^{s-1} N_{1}^{\frac{1}{2}-s+} N_{1}^{3-} \prod_{j=1}^{3}\left\|u_{j}\right\|_{U_{S}^{2}} \\
& \lesssim \delta^{\frac{1}{6}} N^{-\frac{1}{2}+} N_{1}^{3-} \prod_{j=1}^{3}\left\|u_{j}\right\|_{U_{S}^{2}} .
\end{aligned}
$$

Case 2. $N_{1} \sim N_{2} \gg N_{3}, N_{1} \gtrsim N$

We write $M=M_{1}-M_{2}$, where $M_{1}=\sum_{j=1}^{3}\left(\xi_{j}^{3}+\eta_{j}^{3}\right)$ and $M_{2}=\frac{\sum_{j=1}^{3}\left(\xi_{j}^{3}+\eta_{j}^{3}\right) m^{2}\left(\zeta_{j}\right)}{m\left(\zeta_{1}\right) m\left(\zeta_{2}\right) m\left(\zeta_{3}\right)}$. The corresponding terms are

$$
\left.L_{1,1}=\mid \int_{0}^{\delta} \int_{\sum_{j=1}^{3} \zeta_{j}=0} M_{1}\left(\zeta_{1}, \zeta_{2}, \zeta_{3}\right) \prod_{j=1}^{3} \widehat{u_{j}} \zeta_{j}\right) \mid
$$

and

$$
L_{1,2}=\left|\int_{0}^{\delta} \int_{\sum_{j=1}^{3} \zeta_{j}=0} M_{2}\left(\zeta_{1}, \zeta_{2}, \zeta_{3}\right) \prod_{j=1}^{3} \widehat{u_{j}}\left(\zeta_{j}\right)\right| .
$$

Estimate (3.9) tells us that

$$
\begin{aligned}
L_{1,1} & \lesssim \delta^{\frac{1}{2}} N_{1} N_{3}^{\frac{3}{2}} N_{1}^{-2+} N_{3}^{-1+} \prod_{j=1}^{3} N_{j}^{1-}\left\|u_{j}\right\|_{U_{S}^{2}} \\
& \lesssim \delta^{\frac{1}{2}} N^{-\frac{1}{2}+} \prod_{j=1}^{3} N_{j}^{1-}\left\|u_{j}\right\|_{U_{S}^{2}} .
\end{aligned}
$$

Using (3.10), we get the contribution of $L_{1,2}$

$$
\begin{aligned}
L_{1,2} & \lesssim \delta^{\frac{1}{2}} N_{1} N_{3}^{\frac{3}{2}}\left(1 \vee\left(\frac{N_{3}}{N}\right)^{1-s}\right) N_{1}^{-2+} N_{3}^{-1+} \prod_{j=1}^{3} N_{j}^{1-}\left\|u_{j}\right\|_{U_{S}^{2}} \\
& \lesssim \delta^{\frac{1}{2}} N^{-\frac{1}{2}+} \prod_{j=1}^{3} N_{j}^{1-}\left\|u_{j}\right\|_{U_{S}^{2}} .
\end{aligned}
$$

This complete the proof of (3.12), and hence (3.11). 
Proposition 3.8. Let $\frac{5}{7}<s<1$. We have

$$
\left|\int_{0}^{\delta} \Lambda_{4}\left(\left(\xi_{1}+\xi_{2}+\eta_{1}+\eta_{2}\right) \frac{m\left(\zeta_{1}+\zeta_{2}\right)}{m\left(\zeta_{1}\right) m\left(\zeta_{2}\right)} ; I u\right)\right| \lesssim \delta^{\frac{1}{6}} N^{-1+}\|I u\|_{Y^{1}}^{4} .
$$

Proof. As previous discussion, it suffices to show

$$
\begin{aligned}
& \left|\int_{0}^{\delta} \int_{\sum_{j=1}^{4} \lambda_{j}=0}\left[\left(\xi_{1}+\xi_{2}+\eta_{1}+\eta_{2}\right) \frac{m\left(\zeta_{1}+\zeta_{2}\right)}{m\left(\zeta_{1}\right) m\left(\zeta_{2}\right)}\right]_{s y m} \prod_{j=1}^{4} \mathscr{F}\left(\chi_{\delta} u_{j}\right)\left(\lambda_{j}\right)\right| \\
& \lesssim \delta^{\frac{1}{6}} N^{-1+} \prod_{j=1}^{4} N_{j}^{1-}\left\|u_{j}\right\|_{U_{S}^{2}} .
\end{aligned}
$$

If $N \gg N_{j}(j=1,2,3,4)$, then it is easy to see $\left[\left(\xi_{1}+\xi_{2}\right) \frac{m\left(\zeta_{1}+\zeta_{2}\right)}{m\left(\zeta_{1}\right) m\left(\zeta_{2}\right)}\right]_{s y m}=0$, (3.14) holds trivially. We may assume $N_{1} \geqslant N_{2}, N_{3} \geqslant N_{4}$ by symmetry and let $L_{2}$ denote the left hand of (3.14).

From Hörder's inequalities and (2.5), we obtain

$$
\begin{aligned}
& L_{2} \lesssim\left\|\int_{0}^{\delta} \int_{\zeta=\zeta_{1}+\zeta_{2}}(\xi+\eta) \frac{m(\zeta)}{m\left(\zeta_{1}\right) m\left(\zeta_{2}\right)} \widehat{u}_{1}\left(\zeta_{1}\right) \widehat{u}_{2}\left(\zeta_{2}\right) d \zeta_{1} d t\right\|_{L^{2}}\left\|\chi_{\delta} u_{3}\right\|_{L^{4}}\left\|\chi_{\delta} u_{4}\right\|_{L^{4}} \\
& \lesssim \delta^{\frac{1}{3}} N_{1}\left\|\mathscr{F}^{-1} \frac{\widehat{u}_{1}}{m\left(\zeta_{1}\right)}\right\|_{L_{t}^{6} L_{x, y}^{4}}\left\|\mathscr{F}^{-1} \frac{\widehat{u}_{2}}{m\left(\zeta_{2}\right)}\right\|_{L_{t}^{6} L_{x, y}^{4}}\left\|u_{3}\right\|_{L_{t}^{6} L_{x, y}^{4}}\left\|u_{4}\right\|_{L_{t}^{6} L_{x, y}^{4}} \\
& \lesssim \delta^{\frac{1}{3}} N_{1}\left\|\mathscr{F}^{-1} \frac{\widehat{u}_{1}}{m\left(\zeta_{1}\right)}\right\|_{U_{S}^{6}}\left\|\mathscr{F}^{-1} \frac{\widehat{u}_{2}}{m\left(\zeta_{2}\right)}\right\|_{U_{S}^{6}}\left\|u_{3}\right\|_{U_{S}^{6}}\left\|u_{4}\right\|_{U_{S}^{6}} \\
& \lesssim \delta^{\frac{1}{3}} N_{1}\left\|\mathscr{F}^{-1} \frac{\widehat{u}_{1}}{m\left(\zeta_{1}\right)}\right\|_{U_{S}^{2}}\left\|\mathscr{F}^{-1} \frac{\widehat{u}_{2}}{m\left(\zeta_{2}\right)}\right\|_{U_{S}^{2}}\left\|u_{3}\right\|_{U_{S}^{2}}\left\|u_{4}\right\|_{U_{S}^{2}} \\
& \lesssim \delta^{\frac{1}{3}} \frac{N_{1}}{m\left(N_{1}\right) m\left(N_{2}\right)} \prod_{j=1}^{4}\left\|u_{j}\right\|_{U_{S}^{2}} .
\end{aligned}
$$

Case 1. $N_{1} \ll N_{3}$

This implies $N_{3} \sim N_{4} \gg N_{1} \geqslant N_{2}, N_{3} \sim N_{4} \gtrsim N$. Combining the pointwise bound about the symbol

$$
\frac{N_{j}^{-1+}}{m\left(N_{j}\right)} \lesssim 1
$$

for all $N_{j}$, we have

$$
\begin{aligned}
L_{2} & \lesssim \delta^{\frac{1}{3}} N_{1} N_{3}^{-2+} \prod_{j=1}^{4} N_{j}^{1-}\left\|u_{j}\right\|_{U_{S}^{2}} \\
& \lesssim \delta^{\frac{1}{3}} N^{-1+} \prod_{j=1}^{4} N_{j}^{1-}\left\|u_{j}\right\|_{U_{S}^{2}} .
\end{aligned}
$$


Case 2. $N_{1} \gtrsim N_{3}, N_{1} \sim N_{2} \gtrsim N$

Similarly to Case 1, one obtain that

$$
\begin{aligned}
L_{2} & \lesssim \delta^{\frac{1}{3}} \frac{N_{1}}{m\left(N_{1}\right) m\left(N_{2}\right)} N_{1}^{-2+} \prod_{j=1}^{4} N_{j}^{1-}\left\|u_{j}\right\|_{U_{S}^{2}} \\
& \lesssim \delta^{\frac{1}{3}} N_{1}\left(\frac{N_{1}}{N}\right)^{2(1-s)} N_{1}^{-2+} \prod_{j=1}^{4} N_{j}^{1-}\left\|u_{j}\right\|_{U_{S}^{2}} \\
& \lesssim \delta^{\frac{1}{3}} N^{-1+} \prod_{j=1}^{4} N_{j}^{1-}\left\|u_{j}\right\|_{U_{S}^{2}} .
\end{aligned}
$$

Case 3. $N_{1} \gtrsim N_{3}, N_{1} \gg N_{2}$

In this case, $N_{1} \sim N_{3} \gtrsim N$, we control $L_{2}$ by using (3.1),

$$
\begin{aligned}
T_{2} & \lesssim\left\|\int_{\lambda=\lambda_{1}+\lambda_{2}}(\xi+\eta) \frac{m\left(\zeta_{1}+\zeta_{2}\right)}{m\left(\zeta_{1}\right) m\left(\zeta_{2}\right)} \tilde{u}_{1}\left(\lambda_{1}\right) \tilde{u}_{2}\left(\lambda_{2}\right) d \lambda_{1}\right\|_{L_{\lambda}^{2}}\left\|\chi_{\delta} u_{3}\right\|_{L^{4}}\left\|\chi_{\delta} u_{4}\right\|_{L^{4}} \\
& \lesssim \delta^{\frac{1}{6}} \frac{N_{2}^{\frac{1}{2}}}{m\left(N_{2}\right)} \prod_{j=1}^{4}\left\|u_{j}\right\|_{U_{S}^{2}} \\
& \lesssim \delta^{\frac{1}{6}} N_{2}^{\frac{1}{2}}\left(1 \vee\left(\frac{N_{2}}{N}\right)^{1-s}\right) N_{1}^{-2+} N_{2}^{-1+} \prod_{j=1}^{4} N_{j}^{1-}\left\|u_{j}\right\|_{U_{S}^{2}} \\
& \lesssim \delta^{\frac{1}{6}} N^{-2+} \prod_{j=1}^{4} N_{j}^{1-}\left\|u_{j}\right\|_{U_{S}^{2}} .
\end{aligned}
$$

Therefore we complete the proof of (3.14).

It's not hard to obtain the following proposition in a similar way.

Proposition 3.9. Let $\frac{5}{7}<s<1$. We have

$$
\left|\int_{0}^{\delta} \Lambda_{3}\left(\left(\xi_{3}+\eta_{3}\right) \frac{m\left(\zeta_{1}+\zeta_{2}\right)}{m\left(\zeta_{1}\right) m\left(\zeta_{2}\right)} ; I u\right)\right| \lesssim \delta^{\frac{2}{3}} N^{-2+}\|I u\|_{Y^{1}}^{3} .
$$

Proposition 3.10. Let $\frac{5}{7}<s<1, N \gg 1$. Assume $u_{0}$ satisfies $\left|E\left(I u_{0}\right)\right| \leqslant 1$. Then there is a constant $\delta=\delta\left(\left\|u_{0}\right\|_{L^{2}\left(\mathbb{R}^{2}\right)}\right)>0$ so that there exists a unique solution

$$
u(x, y, t) \in C\left([0, \delta], H^{s}\left(\mathbb{R}^{2}\right)\right)
$$

of (1.1) satisfying

$$
E\left(I_{N} u\right)(\delta)=E\left(I_{N} u\right)(0)+\delta^{\frac{1}{6}} O\left(N^{-\frac{1}{2}+}\right)
$$


Proof. From Proposition 3.5, there exsits a unique solution $u$ to (1.1) on $[0, \delta]$ satisfying $\|I u\|_{Y^{1}} \lesssim 1$.

Combining (3.7), Proposition 3.7 and Proposition 3.8, one has

$$
\begin{aligned}
|E(I u)(\delta)-E(I u)(0)| & =\mid \int_{0}^{\delta} \Lambda_{3}\left(\left(\xi_{1}^{3}+\eta_{1}^{3}\right)\left(1-\frac{m\left(\zeta_{2}+\zeta_{3}\right)}{m\left(\zeta_{2}\right) m\left(\zeta_{3}\right)}\right) ; I u\right) \\
& +\int_{0}^{\delta} \Lambda_{4}\left(\left(\xi_{1}+\xi_{2}+\eta_{1}+\eta_{2}\right) \frac{m\left(\zeta_{1}+\zeta_{2}\right)}{m\left(\zeta_{1}\right) m\left(\zeta_{2}\right)} ; I u\right) \mid \\
& \lesssim \delta^{\frac{1}{6}} N^{-\frac{1}{2}+}\|I u\|_{Y^{1}}^{3}+\delta^{\frac{1}{6}} N^{-1+}\|I u\|_{Y^{1}}^{4} \\
& \lesssim \delta^{\frac{1}{6}} N^{-\frac{1}{2}+} .
\end{aligned}
$$

Our purpose is to construct a solution $u$ on $[0, T]$ for any $T>0$. Note that $u$ is a solution to (1.1), then $u_{\lambda}(x, y, t)=\lambda^{2} u\left(\lambda x, \lambda y, \lambda^{3} t\right)$ is also the solution to (1.1). Hence it suffices to acquire the well-posedness for $u_{\lambda}$ on $\left[0, \frac{T}{\lambda^{3}}\right]$.

From Proposition 3.1 the energy will be arbitrarily small by taking $\lambda$ small,

$$
\begin{aligned}
E\left(I_{N} u_{\lambda, 0}\right) & \lesssim N^{2(1-s)}\left\|u_{\lambda, 0}\right\|_{\dot{H}^{s}\left(\mathbb{R}^{2}\right)}^{2}+\left\|u_{\lambda, 0}\right\|_{L^{3}\left(\mathbb{R}^{2}\right)}^{3} \\
& \lesssim N^{2(1-s)} \lambda^{2(s+1)}\left\|u_{0}\right\|_{\dot{H}^{s}\left(\mathbb{R}^{2}\right)}^{2}+\lambda^{4}\left\|u_{0}\right\|_{L^{3}\left(\mathbb{R}^{2}\right)}^{3} \\
& \lesssim\left(N^{2(1-s)} \lambda^{2(s+1)}+\lambda^{4}\right)\left(1+\left\|u_{0}\right\|_{H^{s}\left(\mathbb{R}^{2}\right)}\right)^{3}
\end{aligned}
$$

Assume $N \gg 1$ is given ( $N$ will be chose later), then we have

$$
E\left(I_{N} u_{\lambda, 0}\right) \leqslant \frac{1}{4}
$$

by setting

$$
\lambda=\lambda\left(N,\left\|u_{0}\right\|_{H^{s}\left(\mathbb{R}^{2}\right)}\right) \sim N^{\frac{s-1}{s+1}} .
$$

Now applying Proposition 3.10 to $u_{\lambda, 0}$, one gets

$$
E\left(I u_{\lambda}\right)(\delta) \leqslant \frac{1}{4}+C N^{-\frac{1}{2}+}<\frac{1}{2} .
$$

Thus from Proposition 3.5 the solution $u_{\lambda}$ can be extended to $t \in[0,2 \delta]$. Iterating this procedure $M$ steps, we have

$$
E\left(I u_{\lambda}\right)(t) \leqslant \frac{1}{4}+C M N^{-\frac{1}{2}+}
$$

for $t \in[0,(M+1) \delta]$. That's to say, as long as $M N^{-\frac{1}{2}+} \lesssim 1$ the solution $u_{\lambda}$ can be extended to $t \in[0,(M+1) \delta]$. Taking $N(T) \sim T^{\frac{2(s+1)}{7 s-5}+} \gg 1$, then

$$
(M+1) \delta \sim N^{\frac{1}{2}-} \delta \sim T N^{\frac{3(1-s)}{s+1}} \sim \frac{T}{\lambda^{3}} .
$$


Hence, we obtain global well-posedness for (1.1) when $s>\frac{5}{7}$. Moreover, from Proposition 3.1 we have

$$
\begin{aligned}
\|u(T)\|_{H^{s}\left(\mathbb{R}^{2}\right)} & \lesssim \lambda^{-s-1}\left\|u_{\lambda}\left(\frac{T}{\lambda^{3}}\right)\right\|_{H^{s}\left(\mathbb{R}^{2}\right)} \\
& \lesssim \lambda^{-s-1}\left(\left|E\left(I u_{\lambda}\right)\left(\frac{T}{\lambda^{3}}\right)\right|^{\frac{1}{2}}+\left\|u_{\lambda, 0}\right\|_{L^{2}\left(\mathbb{R}^{2}\right)}+\left\|u_{\lambda, 0}\right\|_{L^{2}\left(\mathbb{R}^{2}\right)}^{2}\right) \\
& \lesssim \lambda^{-s-1}\left(1+\left\|u_{0}\right\|_{H^{s}\left(\mathbb{R}^{2}\right)}\right)^{2} \\
& \lesssim T^{\frac{2(1-s)(1+s)}{7 s-5}}-\left(1+\left\|u_{0}\right\|_{H^{s}\left(\mathbb{R}^{2}\right)}\right)^{2}
\end{aligned}
$$

\section{Global attractor}

This section follows Tsugawa's idea which he applied to show the existence of global attarctor for $\mathrm{KdV}$ equation on Sobolev spaces of negative index (see [27] ).

We find a representation of the rescaled equation associated to (1.4)

$$
\left\{\begin{array}{l}
\partial_{t} v+\left(\partial_{x}^{3}+\partial_{y}^{3}\right) v+\left(\partial_{x}+\partial_{y}\right) v^{2}+\gamma \lambda^{-3} v=\lambda^{-3} g \\
v(x, y, 0)=v_{0}(x, y) \in H^{s}\left(\mathbb{R}^{2}\right)
\end{array}\right.
$$

with $v(x, y, t)=\lambda^{-2} u\left(\lambda^{-1} x, \lambda^{-1} y, \lambda^{-3} t\right), v_{0}(x, y)=\lambda^{-2} u_{0}\left(\lambda^{-1} x, \lambda^{-1} y\right)$ and $g(x, y)=$ $\lambda^{-2} f\left(\lambda^{-1} x, \lambda^{-1} y\right)$.

From the definition of rescaled operator $I^{\prime}$, one clearly knows

$$
\left\|I^{\prime} v\right\|_{L^{2}}=\lambda^{-1}\|I u\|_{L^{2}}, \quad\left\|I^{\prime} g\right\|_{L^{2}}=\lambda^{-1}\|I f\|_{L^{2}},
$$

and

$$
\left\|I^{\prime} v\right\|_{\dot{H}^{1}}=\lambda^{-2}\|I u\|_{\dot{H}^{1}}, \quad\left\|I^{\prime} g\right\|_{\dot{H}^{1}}=\lambda^{-2}\|I f\|_{\dot{H}^{1}} .
$$

Now, we give the time local result for weakly damped forced ZK equation.

Proposition 4.1. Let $\frac{5}{7}<s<1$. Assume $I^{\prime} v_{0} \in H^{1}\left(\mathbb{R}^{2}\right)$ and $I^{\prime} g \in H^{1}\left(\mathbb{R}^{2}\right)$, then there is a constant $\delta=\delta\left(\left\|I^{\prime} v_{0}\right\|_{H^{1}\left(\mathbb{R}^{2}\right)}, \lambda^{-3}\left\|I^{\prime} g\right\|_{H^{1}\left(\mathbb{R}^{2}\right)}, \gamma \lambda^{-3}\right)>0$ so that there exists a unique solution

$$
v(x, y, t) \in C\left([0, \delta], H^{s}\left(\mathbb{R}^{2}\right)\right)
$$

of (4.1) satisfying

$$
\left\|I^{\prime} v\right\|_{Y^{1}} \lesssim\left\|I^{\prime} v_{0}\right\|_{H^{1}\left(\mathbb{R}^{2}\right)}+\lambda^{-3}\left\|I^{\prime} g\right\|_{H^{1}\left(\mathbb{R}^{2}\right)}
$$

and

$$
\sup _{t \in[0, \delta]}\left\|I^{\prime} v(t)\right\|_{H^{1}} \lesssim\left\|I^{\prime} v_{0}\right\|_{H^{1}\left(\mathbb{R}^{2}\right)}+\lambda^{-3}\left\|I^{\prime} g\right\|_{H^{1}\left(\mathbb{R}^{2}\right)} .
$$


Proof. Acting $I^{\prime}$ on (4.1) gives

$$
\partial_{t} I^{\prime} v+\left(\partial_{x}^{3}+\partial_{y}^{3}\right) I^{\prime} v+\left(\partial_{x}+\partial_{y}\right) I^{\prime} v^{2}+\gamma \lambda^{-3} I^{\prime} v=\lambda^{-3} I^{\prime} g
$$

which also can been written as an intergral equation

$$
I^{\prime} v=\mathscr{T} I^{\prime} v
$$

where

$$
\mathscr{T} I^{\prime} v=\chi\left(\frac{t}{\delta}\right) e^{t S} I v_{0}-\int_{0}^{t} e^{\left(t-t^{\prime}\right) S} \chi\left(\frac{t}{\delta}\right)\left(\left(\partial_{x}+\partial_{y}\right) I^{\prime} v^{2}+\gamma \lambda^{-3} I^{\prime} v-\lambda^{-3} I^{\prime} g\right) d t^{\prime} .
$$

By the duality of $U^{p}$, we have

$$
\begin{aligned}
& \left\|\int_{0}^{t} e^{\left(t-t^{\prime}\right) S} \chi\left(\frac{t}{\delta}\right)\left(\gamma \lambda^{-3} I^{\prime} v-\lambda^{-3} I^{\prime} g\right) d t^{\prime}\right\|_{Y^{1}} \\
\lesssim & \left(\sum_{N_{1}} N_{1}^{2}\left(\sup _{\|w\|_{V_{S}^{2}} \leqslant 1}\left|\int_{\mathbb{R}^{3}} \chi\left(\frac{t}{\delta}\right)\left(\gamma \lambda^{-3} P_{N_{1}} I^{\prime} v-\lambda^{-3} P_{N_{1}} I^{\prime} g\right) w d x d y d t\right|\right)^{2}\right)^{\frac{1}{2}} \\
\lesssim & \left(\sum_{N_{1}} N_{1}^{2}\left(\sup _{\|w\|_{V_{S}^{2}} \leqslant 1}\left\|\gamma \lambda^{-3} P_{N_{1}} I^{\prime} v-\lambda^{-3} P_{N_{1}} I^{\prime} g\right\|_{L_{t}^{\infty} L_{x, y}^{2}}\left\|\chi\left(\frac{t}{\delta}\right) w\right\|_{L_{t}^{1} L_{x, y}^{2}}\right)^{2}\right)^{\frac{1}{2}} \\
\lesssim & \delta\left(\sum_{N_{1}} N_{1}^{2}\left(\left\|\gamma \lambda^{-3} P_{N_{1}} I^{\prime} v\right\|_{U_{S}^{2}}+\lambda^{-3}\left\|P_{N_{1}} I^{\prime} g\right\|_{L_{x, y}^{2}}\right)^{2}\right)^{\frac{1}{2}} \\
\lesssim & \delta\left(\gamma \lambda^{-3}\left\|I^{\prime} v\right\|_{Y^{1}}+\lambda^{-3}\left\|I^{\prime} g\right\|_{H^{1}}\right) .
\end{aligned}
$$

Set

$$
B=\left\{I^{\prime} v \in Y^{1} \mid\left\|I^{\prime} v\right\|_{Y^{1}}<C_{0}\left(\left\|I^{\prime} v_{0}\right\|_{H^{1}}+\lambda^{-3}\left\|I^{\prime} g\right\|_{H^{1}}\right)\right\} .
$$

Applying Proposition 3.4 and (4.3), on $B$ one gets

$$
\begin{aligned}
\left\|\mathscr{T} I^{\prime} v\right\|_{Y^{1}} & \lesssim\left\|I^{\prime} v_{0}\right\|_{H^{1}}+\delta^{\frac{1}{6}}\left\|I^{\prime} v\right\|_{Y^{1}}^{2}+\delta \gamma \lambda^{-3}\left\|I^{\prime} v\right\|_{Y^{1}}+\delta \lambda^{-3}\left\|I^{\prime} g\right\|_{H^{1}} \\
& \lesssim \delta^{\frac{1}{6}} C_{0}^{2}\left(\left\|I^{\prime} v_{0}\right\|_{H^{1}}+\lambda^{-3}\left\|I^{\prime} g\right\|_{H^{1}}\right)^{2}+\left(1+C_{0} \delta \gamma \lambda^{-3}\right)\left(\left\|I^{\prime} v_{0}\right\|_{H^{1}}+\lambda^{-3}\left\|I^{\prime} g\right\|_{H^{1}}\right),
\end{aligned}
$$

and

$$
\begin{aligned}
\left\|\mathscr{T} I^{\prime} v_{1}-\mathscr{T} I^{\prime} v_{2}\right\|_{Y^{1}} & \lesssim\left(\delta \gamma \lambda^{-3}+\delta^{\frac{1}{6}}\left\|I^{\prime} v_{1}\right\|_{Y^{1}}+\delta^{\frac{1}{6}}\left\|I^{\prime} v_{2}\right\|_{Y^{1}}\right)\left\|I^{\prime} v_{1}-I^{\prime} v_{2}\right\|_{Y^{1}} \\
& \lesssim\left(\delta \gamma \lambda^{-3}+\delta^{\frac{1}{6}} C_{0}\left(\left\|I^{\prime} v_{0}\right\|_{H^{1}}+\lambda^{-3}\left\|I^{\prime} g\right\|_{H^{1}}\right)\right)\left\|I^{\prime} v_{1}-I^{\prime} v_{2}\right\|_{Y^{1}}
\end{aligned}
$$

If we assume

$$
\lambda^{-3} \gamma \ll 1, \quad\left\|I^{\prime} v_{0}\right\|_{H^{1}} \ll 1, \quad \lambda^{-3}\left\|I^{\prime} g\right\|_{H^{1}} \ll 1
$$

then

$$
\mathscr{T}: B \rightarrow B
$$


is a strict contraction mapping.

Finally, fixing $\lambda=\lambda_{0}$ and $I^{\prime} v_{0}, I^{\prime} g \in H^{1}$, we consider $\sigma$-scaling of $v$

$$
w(x, y, t)=\sigma^{-2} v\left(\sigma^{-1} x, \sigma^{-1} y, \sigma^{-3} t\right) .
$$

It's equivalent to consider well-posedness on $\left[0, \sigma^{3} \delta\right]$ for $w$.

Observe that

$$
\begin{gathered}
\left(\sigma \lambda_{0}\right)^{-3} \gamma \ll 1 \\
\left\|I^{\prime \prime} w_{0}\right\|_{H^{1}} \lesssim \sigma^{-1}\left\|I^{\prime} v_{0}\right\|_{H^{1}} \ll 1, \\
\left(\sigma \lambda_{0}\right)^{-3}\left\|I^{\prime} g\right\|_{H^{1}} \ll 1
\end{gathered}
$$

provided $\sigma$ is chosen to be sufficiently large, which verifies (4.4). It means that Cauchy problem for $w$ is well-posed on the time interval $[0,1]$. Hence, (4.2) is locally well-posed on $\left[0, \sigma^{-3}\right]$. We complete the proof.

In the next place, we explore the increment of $I^{\prime} v$ through modified energy $E\left(I^{\prime} v\right)$.

From (4.2), we obtain

$$
\begin{aligned}
\frac{d E\left(I^{\prime} v\right)(t)}{d t}= & -\int_{\mathbb{R}^{2}}\left(\Delta I^{\prime} v-\partial_{x} \partial_{y} I^{\prime} v+\left(I^{\prime} v\right)^{2}\right) \partial_{t} I^{\prime} v d x d y \\
= & \int_{\mathbb{R}^{2}}\left(\left(\partial_{x}^{3}+\partial_{y}^{3}\right) I^{\prime} v+\left(\partial_{x}+\partial_{y}\right) I^{\prime} v^{2}\right)\left(\left(\Delta-\partial_{x} \partial_{y}\right) I^{\prime} v+\left(I^{\prime} v\right)^{2}\right) d x d y \\
& +\int_{\mathbb{R}^{2}}\left(\gamma \lambda^{-3} I^{\prime} v-\lambda^{-3} I^{\prime} g\right)\left(\left(\Delta-\partial_{x} \partial_{y}\right) I^{\prime} v+\left(I^{\prime} v\right)^{2}\right) d x d y \\
= & -2 \gamma \lambda^{-3} E\left(I^{\prime} v\right)-\int_{\mathbb{R}^{2}} \lambda^{-3} I^{\prime} g\left(\left(\Delta-\partial_{x} \partial_{y}\right) I^{\prime} v+\left(I^{\prime} v\right)^{2}\right) d x d y \\
& +\frac{1}{3} \gamma \lambda^{-3} \int_{\mathbb{R}^{2}}\left(I^{\prime} v\right)^{3} d x d y+\Lambda_{3}\left(\left(\xi_{1}^{3}+\eta_{1}^{3}\right)\left(1-\frac{m\left(\zeta_{2}+\zeta_{3}\right)}{m\left(\zeta_{2}\right) m\left(\zeta_{3}\right)}\right) ; I^{\prime} v\right) \\
& +\Lambda_{4}\left(\left(\xi_{1}+\xi_{2}+\eta_{1}+\eta_{2}\right) \frac{m\left(\zeta_{1}+\zeta_{2}\right)}{m\left(\zeta_{1}\right) m\left(\zeta_{2}\right)} ; I^{\prime} v\right)
\end{aligned}
$$

This implies

$$
\begin{aligned}
\frac{d}{d t} E\left(I^{\prime} v\right)(t) e^{2 \gamma \lambda^{-3} t}= & -\int_{\mathbb{R}^{2}} \lambda^{-3} I^{\prime} g\left(\left(\Delta-\partial_{x} \partial_{y}\right) I^{\prime} v+\left(I^{\prime} v\right)^{2}\right) e^{2 \gamma \lambda^{-3} t} d x d y \\
& +\frac{1}{3} \gamma \lambda^{-3}\left\|I^{\prime} v\right\|_{L^{3}}^{3} e^{2 \gamma \lambda^{-3} t}+\left(\Lambda_{3}+\Lambda_{4}\right) e^{2 \gamma \lambda^{-3} t}
\end{aligned}
$$

Integrating (4.5) over $\left[0, T^{\prime}\right]$, one gets

$$
\begin{aligned}
& E\left(I^{\prime} v\right)\left(T^{\prime}\right) e^{2 \gamma \lambda^{-3} T^{\prime}}-E\left(I^{\prime} v\right)(0) \\
= & -\int_{0}^{T^{\prime}} \int_{\mathbb{R}^{2}} \lambda^{-3} I^{\prime} g\left[\left(\Delta-\partial_{x} \partial_{y}\right) I^{\prime} v+\left(I^{\prime} v\right)^{2}\right] e^{2 \gamma \lambda^{-3} t} d x d y d t \\
& +\frac{1}{3} \gamma \lambda^{-3} \int_{0}^{T^{\prime}}\left\|I^{\prime} v\right\|_{L^{3}}^{3} e^{2 \gamma \lambda^{-3} t} d t+\int_{0}^{T^{\prime}}\left(\Lambda_{3}+\Lambda_{4}\right) e^{2 \gamma \lambda^{-3} t} d t .
\end{aligned}
$$


Lemma 4.2. Assume that $v$ is a solution of (4.1) on $\left[0, T^{\prime}\right]$. Then, we have

$$
\begin{aligned}
& \sup _{t \in\left[0, T^{\prime}\right]}\left\|I^{\prime} v(t)\right\|_{L^{2}}^{2} e^{2 \gamma \lambda^{-3} t} \\
& \leqslant C_{1}\left(\left\|I^{\prime} v_{0}\right\|_{L^{2}}^{2}+\frac{1}{\gamma^{2}}\left\|I^{\prime} g\right\|_{L^{2}}^{2} e^{2 \gamma \lambda^{-3} T^{\prime}}+\left|\int_{0}^{T^{\prime}} e^{2 \gamma \lambda^{-3} t} \Lambda_{3}^{\prime} d t\right|\right),
\end{aligned}
$$

and

$$
\begin{aligned}
& \sup _{t \in\left[0, T^{\prime}\right]}\left\|I^{\prime} v(t)\right\|_{\dot{H}^{1}}^{2} e^{2 \gamma \lambda^{-3} t} \\
& \leqslant C_{1}\left(\left\|I^{\prime} v_{0}\right\|_{\dot{H}^{1}}^{2}+\left\|I^{\prime} v_{0}\right\|_{L^{2}}^{4}+\frac{1}{\gamma^{2}}\left\|I^{\prime} g\right\|_{\dot{H}^{1}}^{2} e^{2 \gamma \lambda^{-3} T^{\prime}}+\frac{1}{\gamma^{4}}\left\|I^{\prime} g\right\|_{L^{2}}^{4} e^{2 \gamma \lambda^{-3} T^{\prime}}\right. \\
& \left.\quad+\left|\int_{0}^{T^{\prime}}\left(\Lambda_{3}+\Lambda_{4}\right) e^{2 \gamma \lambda^{-3} t} d t\right|+\left|\int_{0}^{T^{\prime}} \Lambda_{3}^{\prime} e^{2 \gamma \lambda^{-3} t} d t\right|^{2}\right),
\end{aligned}
$$

where $\Lambda_{3}^{\prime}=\Lambda_{3}\left(\left(\xi_{3}+\eta_{3}\right) \frac{m\left(\zeta_{1}+\zeta_{2}\right)}{m\left(\zeta_{1}\right) m\left(\zeta_{2}\right)} ; I^{\prime} v\right), C_{1}>1$.

Proof. Similarly to (4.5), one gets

$$
\frac{d}{d t}\left\|I^{\prime} v(t)\right\|_{L^{2}\left(\mathbb{R}^{2}\right)}^{2} e^{2 \gamma \lambda^{-3} t}=2 \lambda^{-3} e^{2 \gamma \lambda^{-3} t} \int_{\mathbb{R}^{2}} I^{\prime} g I^{\prime} v d x d y-2 e^{2 \gamma \lambda^{-3} t} \Lambda_{3}^{\prime}
$$

which implies

$$
\begin{aligned}
& \left\|I^{\prime} v(t)\right\|_{L^{2}\left(\mathbb{R}^{2}\right)}^{2} e^{2 \gamma \lambda^{-3} t} \\
\lesssim & \left\|I^{\prime} v_{0}\right\|_{L^{2}}^{2}+\left|\int_{0}^{T^{\prime}} e^{2 \gamma \lambda^{-3} t} \Lambda_{3}^{\prime} d t\right|+\frac{e^{\gamma \lambda^{-3} T^{\prime}}}{\gamma}\left\|I^{\prime} g\right\|_{L^{2}} \sup _{t \in\left[0, T^{\prime}\right]}\left\|I^{\prime} v(t)\right\|_{L^{2}} e^{\gamma \lambda^{-3} t} \\
\lesssim & \left\|I^{\prime} v_{0}\right\|_{L^{2}}^{2}+\left|\int_{0}^{T^{\prime}} e^{2 \gamma \lambda^{-3} t} \Lambda_{3}^{\prime} d t\right|+\frac{C_{\epsilon}}{\gamma^{2}} e^{2 \gamma \lambda^{-3} T^{\prime}}\left\|I^{\prime} g\right\|_{L^{2}}^{2}+\epsilon \sup _{t \in\left[0, T^{\prime}\right]}\left\|I^{\prime} v(t)\right\|_{L^{2}}^{2} e^{2 \gamma \lambda^{-3} t}
\end{aligned}
$$

for $t \in\left[0, T^{\prime}\right]$.

As the last term of (4.9) can be absorbed by the left side via taking $\epsilon$ sufficiently small, we obtain (4.7).

Using Hörder's inequalities, one has

$$
\begin{aligned}
& \left|\int_{0}^{T^{\prime}} \int_{\mathbb{R}^{2}} \lambda^{-3} I^{\prime} g\left(\left(\Delta-\partial_{x} \partial_{y}\right) I^{\prime} v+\left(I^{\prime} v\right)^{2}\right) e^{2 \gamma \lambda^{-3} t} d x d y d t\right| \\
& \lesssim \frac{e^{\gamma \lambda^{-3} T^{\prime}}}{\gamma}\left\|\nabla I^{\prime} g\right\|_{L^{2}} \sup _{t \in\left[0, T^{\prime}\right]}\left\|\nabla I^{\prime} v(t)\right\|_{L^{2}} e^{\gamma \lambda^{-3} t} \\
& \quad+\frac{e^{\frac{2}{3} \gamma \lambda^{-3} T^{\prime}}}{\gamma}\left\|I^{\prime} g\right\|_{L^{3}} \sup _{t \in\left[0, T^{\prime}\right]}\left\|I^{\prime} v(t)\right\|_{L^{3}}^{2} e^{\frac{4}{3} \gamma \lambda^{-3} t}
\end{aligned}
$$




$$
\begin{aligned}
\lesssim & \frac{C_{\epsilon} e^{2 \gamma \lambda^{-3} T^{\prime}}}{\gamma^{2}}\left\|I^{\prime} g\right\|_{\dot{H}^{1}}^{2}+\epsilon \sup _{t \in\left[0, T^{\prime}\right]}\left\|I^{\prime} v(t)\right\|_{\dot{H}^{1}}^{2} e^{2 \gamma \lambda^{-3} t} \\
& +\frac{e^{2 \gamma \lambda^{-3} T^{\prime}}}{\gamma^{3}}\left\|I^{\prime} g\right\|_{L^{3}}^{3}+\sup _{t \in\left[0, T^{\prime}\right]}\left\|I^{\prime} v(t)\right\|_{L^{3}}^{3} e^{2 \gamma \lambda^{-3} t} .
\end{aligned}
$$

Hence, from (4.6) and (4.10) we obtain

$$
\begin{aligned}
\left\|I^{\prime} v(t)\right\|_{\dot{H}^{1}}^{2} e^{2 \gamma \lambda^{-3} t} \lesssim\left\|I^{\prime} v_{0}\right\|_{\dot{H}^{1}}^{2}+\frac{e^{2 \gamma \lambda^{-3} T^{\prime}}}{\gamma^{2}}\left\|I^{\prime} g\right\|_{\dot{H}^{1}}^{2}+\left|\int_{0}^{T^{\prime}}\left(\Lambda_{3}+\Lambda_{4}\right) e^{2 \gamma \lambda^{-3} t} d t\right| \\
+\frac{e^{2 \gamma \lambda^{-3} T^{\prime}}}{\gamma^{3}}\left\|I^{\prime} g\right\|_{L^{3}}^{3}+\sup _{t \in\left[0, T^{\prime}\right]}\left\|I^{\prime} v(t)\right\|_{L^{3}}^{3} e^{2 \gamma \lambda^{-3} t}
\end{aligned}
$$

for $t \in\left[0, T^{\prime}\right]$.

Gagliardo-Nirenberg's inequality and (4.7) give

$$
\begin{aligned}
\left\|I^{\prime} g\right\|_{L^{3}}^{3} & \lesssim\left\|I^{\prime} g\right\|_{\dot{H}^{1}}\left\|I^{\prime} g\right\|_{L^{2}}^{2} \\
& \lesssim \gamma\left\|I^{\prime} g\right\|_{\dot{H}^{1}}^{2}+\frac{1}{\gamma}\left\|I^{\prime} g\right\|_{L^{2}}^{4}
\end{aligned}
$$

and

$$
\begin{aligned}
& \sup _{t \in\left[0, T^{\prime}\right]}\left\|I^{\prime} v\right\|_{L^{3}}^{3} e^{2 \gamma \lambda^{-3} t} \\
& \lesssim \sup _{t \in\left[0, T^{\prime}\right]}\left\|I^{\prime} v\right\|_{\dot{H}^{1}}\left\|I^{\prime} v\right\|_{L^{2}}^{2} e^{2 \gamma \lambda^{-3} t} \\
& \lesssim C_{\epsilon} \sup _{t \in\left[0, T^{\prime}\right]}\left\|I^{\prime} v\right\|_{L^{2}}^{4} e^{2 \gamma \lambda^{-3} t}+\epsilon \sup _{t \in\left[0, T^{\prime}\right]}\left\|I^{\prime} v\right\|_{\dot{H}^{1}}^{2} e^{2 \gamma \lambda^{-3} t} \\
& \lesssim C_{\epsilon}\left\|I^{\prime} v_{0}\right\|_{L^{2}}^{4}+\frac{C_{\epsilon}}{\gamma^{4}} e^{2 \gamma \lambda^{-3} T^{\prime}}\left\|I^{\prime} g\right\|_{L^{2}}^{4}+C_{\epsilon}\left|\int_{0}^{T^{\prime}} e^{2 \gamma \lambda^{-3} t} \Lambda_{3}^{\prime} d t\right|^{2} \\
& \quad+\epsilon \sup _{t \in\left[0, T^{\prime}\right]}\left\|I^{\prime} v\right\|_{\dot{H}^{1}}^{2} e^{2 \gamma \lambda^{-3} t} .
\end{aligned}
$$

Collecting (4.11), (4.12) and (4.13), we can get (4.8).

The following a-priori estimate is impactful for controlling $\|u\|_{H^{s}}$.

Proposition 4.3. Let $C_{2} \ll 1, C_{3}>1, C_{4} \gg 1$ and $T^{\prime}>0$ be given. Assume $v$ is a solution of (4.1) on $\left[0, T^{\prime}\right]$. If $\lambda^{3} \geqslant \gamma,\left(N^{\prime}\right)^{\frac{1}{2}-} \geqslant C_{4} \lambda^{2} T^{\prime}$,

$$
\begin{gathered}
1 \lesssim \lambda^{2}\left\|I^{\prime} v_{0}\right\|_{L^{2}}^{2}, \quad 1 \lesssim \lambda^{2}\left\|I^{\prime} g\right\|_{L^{2}}^{2}, \\
\left\|I^{\prime} v_{0}\right\|_{L^{2}}^{2}+\frac{1}{\gamma^{2}}\left\|I^{\prime} g\right\|_{L^{2}}^{2} e^{2 \gamma \lambda^{-3} T^{\prime}} \leqslant C_{2}, \\
\left\|I^{\prime} v_{0}\right\|_{\dot{H}^{1}}^{2}+\frac{1}{\gamma^{2}}\left\|I^{\prime} g\right\|_{\dot{H}^{1}}^{2} e^{2 \gamma \lambda^{-3} T^{\prime}} \leqslant \lambda^{-2} C_{2},
\end{gathered}
$$


then we have

$$
\begin{gathered}
\left\|I^{\prime} v\left(T^{\prime}\right)\right\|_{L^{2}}^{2} e^{2 \gamma \lambda^{-3} T^{\prime}} \leqslant C_{3}\left(\left\|I^{\prime} v_{0}\right\|_{L^{2}}^{2}+\frac{1}{\gamma^{2}}\left\|I^{\prime} g\right\|_{L^{2}}^{2} e^{2 \gamma \lambda^{-3} T^{\prime}}\right), \\
\left\|I^{\prime} v\left(T^{\prime}\right)\right\|_{\dot{H}^{1}}^{2} e^{2 \gamma \lambda^{-3} T^{\prime}} \leqslant C_{3}\left(\left\|I^{\prime} v_{0}\right\|_{\dot{H}^{1}}^{2}+\left\|I^{\prime} v_{0}\right\|_{L^{2}}^{4}+\frac{1}{\gamma^{2}}\left\|I^{\prime} g\right\|_{\dot{H}^{1}}^{2} e^{2 \gamma \lambda^{-3} T^{\prime}}\right. \\
\left.+\frac{1}{\gamma^{4}}\left\|I^{\prime} g\right\|_{L^{2}}^{4} e^{2 \gamma \lambda^{-3} T^{\prime}}\right) .
\end{gathered}
$$

Proof. Actually choosing $\lambda$ sufficiently large, from scaling we have

$$
\begin{aligned}
& \left\|I^{\prime} v_{0}\right\|_{\dot{H}^{1}}^{2}+\left\|I^{\prime} v_{0}\right\|_{L^{2}}^{4}+\frac{1}{\gamma^{2}}\left\|I^{\prime} g\right\|_{\dot{H}^{1}}^{2} e^{2 \gamma \lambda^{-3} T^{\prime}}+\frac{1}{\gamma^{4}}\left\|I^{\prime} g\right\|_{L^{2}}^{4} e^{2 \gamma \lambda^{-3} T^{\prime}} \\
& \ll\left\|I^{\prime} v_{0}\right\|_{L^{2}}^{2}+\frac{1}{\gamma^{2}}\left\|I^{\prime} g\right\|_{L^{2}}^{2} e^{2 \gamma \lambda^{-3} T^{\prime}} .
\end{aligned}
$$

Taking $\delta=\delta\left(\left\|I^{\prime} v_{0}\right\|_{H^{1}}, \lambda^{-3}\left\|I^{\prime} g\right\|_{H^{1}}, \gamma \lambda^{-3}\right)$ as in Proposition 4.1 and $j \in \mathbb{N}$ satisfying $\delta j=T^{\prime}$. For $0 \leqslant k \leqslant j, k \in \mathbb{N}$, we prove

$$
\begin{aligned}
& \left\|I^{\prime} v(k \delta)\right\|_{L^{2}}^{2} e^{2 \gamma \lambda^{-3} k \delta} \\
\leqslant & 2 C_{1}\left(\left\|I^{\prime} v_{0}\right\|_{L^{2}}^{2}+\frac{1}{\gamma^{2}}\left\|I^{\prime} g\right\|_{L^{2}}^{2} e^{2 \gamma \lambda^{-3} k \delta}\right) \\
\leqslant & 2 C_{1} C_{2}
\end{aligned}
$$

and

$$
\begin{aligned}
& \left\|I^{\prime} v(k \delta)\right\|_{\dot{H}^{1}}^{2} e^{2 \gamma \lambda^{-3} k \delta} \\
\leqslant & 2 C_{1}\left(\left\|I^{\prime} v_{0}\right\|_{\dot{H}^{1}}^{2}+\left\|I^{\prime} v_{0}\right\|_{L^{2}}^{4}+\frac{1}{\gamma^{2}}\left\|I^{\prime} g\right\|_{\dot{H}^{1}}^{2} e^{2 \gamma \lambda^{-3} k \delta}\right. \\
& \left.+\frac{1}{\gamma^{4}}\left\|I^{\prime} g\right\|_{L^{2}}^{4} e^{2 \gamma \lambda^{-3} k \delta}\right) \\
\leqslant & 4 C_{1} C_{2}
\end{aligned}
$$

by induction.

For $k=0$, (4.14) and (4.15) hold trivially. We assume (4.14) and (4.15) hold true for $k=l$ where $0 \leqslant l \leqslant j-1$. From Lemma 4.2, one has

$$
\begin{aligned}
& \left\|I^{\prime} v((l+1) \delta)\right\|_{L^{2}}^{2} e^{2 \gamma \lambda^{-3}(l+1) \delta} \\
\leqslant & C_{1}\left(\left\|I^{\prime} v_{0}\right\|_{L^{2}}^{2}+\frac{1}{\gamma^{2}}\left\|I^{\prime} g\right\|_{L^{2}}^{2} e^{2 \gamma \lambda^{-3}(l+1) \delta}+\left|\int_{0}^{(l+1) \delta} e^{2 \gamma \lambda^{-3} t} \Lambda_{3}^{\prime} d t\right|\right)
\end{aligned}
$$

and

$$
\left\|I^{\prime} v((l+1) \delta)\right\|_{\dot{H}^{1}}^{2} e^{2 \gamma \lambda^{-3}(l+1) \delta}
$$




$$
\begin{aligned}
\leqslant & C_{1}\left(\left\|I^{\prime} v_{0}\right\|_{\dot{H}^{1}}^{2}+\left\|I^{\prime} v_{0}\right\|_{L^{2}}^{4}+\frac{1}{\gamma^{2}}\left\|I^{\prime} g\right\|_{\dot{H}^{1}}^{2} e^{2 \gamma \lambda^{-3}(l+1) \delta}\right. \\
& +\frac{1}{\gamma^{4}}\left\|I^{\prime} g\right\|_{L^{2}}^{4} e^{2 \gamma \lambda^{-3}(l+1) \delta}+\left|\int_{0}^{(l+1) \delta}\left(\Lambda_{3}+\Lambda_{4}\right) e^{2 \gamma \lambda^{-3} t} d t\right| \\
& \left.+\left|\int_{0}^{(l+1) \delta} \Lambda_{3}^{\prime} e^{2 \gamma \lambda^{-3} t} d t\right|^{2}\right) .
\end{aligned}
$$

Therefore, it suffices to prove

$$
\left|\int_{0}^{(l+1) \delta} e^{2 \gamma \lambda^{-3} t} \Lambda_{3}^{\prime} d t\right| \leqslant\left\|I^{\prime} v_{0}\right\|_{L^{2}}^{2}+\frac{1}{\gamma^{2}}\left\|I^{\prime} g\right\|_{L^{2}}^{2} e^{2 \gamma \lambda^{-3}(l+1) \delta}
$$

and

$$
\begin{aligned}
& \left|\int_{0}^{(l+1) \delta}\left(\Lambda_{3}+\Lambda_{4}\right) e^{2 \gamma \lambda^{-3} t} d t\right|+\left|\int_{0}^{(l+1) \delta} e^{2 \gamma \lambda^{-3} t} \Lambda_{3}^{\prime} d t\right|^{2} \\
\leqslant & \left\|I^{\prime} v_{0}\right\|_{\dot{H}^{1}}^{2}+\left\|I^{\prime} v_{0}\right\|_{L^{2}}^{4}+\frac{1}{\gamma^{2}}\left\|I^{\prime} g\right\|_{\dot{H}^{1}}^{2} e^{2 \gamma \lambda^{-3}(l+1) \delta}+\frac{1}{\gamma^{4}}\left\|I^{\prime} g\right\|_{L^{2}}^{4} e^{2 \gamma \lambda^{-3}(l+1) \delta} .
\end{aligned}
$$

From Proposition 3.9, we have

$$
\begin{aligned}
& \left|\int_{0}^{(l+1) \delta} e^{2 \gamma \lambda^{-3} t} \Lambda_{3}^{\prime} d t\right| \\
= & \left|\int_{0}^{(l+1) \delta} \Lambda_{3}\left(\left(\xi_{3}+\eta_{3}\right) \frac{m\left(\zeta_{1}+\zeta_{2}\right)}{m\left(\zeta_{1}\right) m\left(\zeta_{2}\right)} ; e^{\frac{2}{3} \gamma \lambda^{-3} t} I^{\prime} v\right)\right| \\
\lesssim & \delta^{\frac{2}{3}}\left(N^{\prime}\right)^{-2+} \sum_{m=0}^{l}\left\|e^{\frac{2}{3} \gamma \lambda^{-3} t} I^{\prime} v\right\|_{Y_{[m \delta,(m+1) \delta]}^{1}}^{1} \\
\lesssim & \delta^{\frac{2}{3}}\left(T^{\prime}\right)^{-1} \sum_{m=0}^{l}\left\|I^{\prime} v\right\|_{Y_{[m \delta,(m+1) \delta]}^{1}}^{3} e^{2 \gamma \lambda^{-3}(m+1) \delta} .
\end{aligned}
$$

Proposition 4.1, (4.14) and (4.15) provide the up bound of $\left\|I^{\prime} v\right\|_{Y^{1}}$

$$
\begin{aligned}
& \left\|I^{\prime} v\right\|_{Y_{[m \delta,(m+1) \delta]}^{1}}^{3} e^{2 \gamma \lambda^{-3}(m+1) \delta} \\
\lesssim & \left\|I^{\prime} v(m \delta)\right\|_{H^{1}}^{3} e^{2 \gamma \lambda^{-3}(m+1) \delta}+\left(\lambda^{-3}\left\|I^{\prime} g\right\|_{H^{1}}\right)^{3} e^{2 \gamma \lambda^{-3}(m+1) \delta} \\
\lesssim & \left(C_{1} C_{2}\right)^{\frac{1}{2}} C_{1}\left(\left\|I^{\prime} v_{0}\right\|_{L^{2}}^{2}+\frac{1}{\gamma^{2}}\left\|I^{\prime} g\right\|_{L^{2}}^{2} e^{2 \gamma \lambda^{-3}(m+1) \delta}\right) .
\end{aligned}
$$

Hence, from (4.18) and (4.19), one obtains

$$
\begin{aligned}
& \left|\int_{0}^{(l+1) \delta} e^{2 \gamma \lambda^{-3} t} \Lambda_{3}^{\prime} d t\right| \\
\lesssim & \left(C_{1} C_{2}\right)^{\frac{1}{2}} C_{1} \delta^{\frac{2}{3}}\left(T^{\prime}\right)^{-1}(l+1)\left(\left\|I^{\prime} v_{0}\right\|_{L^{2}}^{2}+\frac{1}{\gamma^{2}}\left\|I^{\prime} g\right\|_{L^{2}}^{2} e^{2 \gamma \lambda^{-3}(l+1) \delta}\right)
\end{aligned}
$$




$$
\lesssim\left(C_{1} C_{2}\right)^{\frac{1}{2}} C_{1} \delta^{-\frac{1}{3}}\left(\left\|I^{\prime} v_{0}\right\|_{L^{2}}^{2}+\frac{1}{\gamma^{2}}\left\|I^{\prime} g\right\|_{L^{2}}^{2} e^{2 \gamma \lambda^{-3}(l+1) \delta}\right) .
$$

We can get (4.16) from (4.20) as $C_{2} \ll 1$.

Moreover,

$$
\begin{aligned}
& \left|\int_{0}^{(l+1) \delta} e^{2 \gamma \lambda^{-3} t} \Lambda_{3}^{\prime} d t\right|^{2} \\
\leqslant & \frac{1}{4}\left(\left\|I^{\prime} v_{0}\right\|_{\dot{H}^{1}}^{2}+\left\|I^{\prime} v_{0}\right\|_{L^{2}}^{4}+\frac{1}{\gamma^{2}}\left\|I^{\prime} g\right\|_{\dot{H}^{1}}^{2} e^{2 \gamma \lambda^{-3}(l+1) \delta}+\frac{1}{\gamma^{4}}\left\|I^{\prime} g\right\|_{L^{2}}^{4} e^{2 \gamma \lambda^{-3}(l+1) \delta}\right)
\end{aligned}
$$

which allows that one only need to show

$$
\begin{aligned}
& \left|\int_{0}^{(l+1) \delta}\left(\Lambda_{3}+\Lambda_{4}\right) e^{2 \gamma \lambda^{-3} t} d t\right| \\
\leqslant & \frac{1}{2}\left(\left\|I^{\prime} v_{0}\right\|_{\dot{H}^{1}}^{2}+\left\|I^{\prime} v_{0}\right\|_{L^{2}}^{4}+\frac{1}{\gamma^{2}}\left\|I^{\prime} g\right\|_{\dot{H}^{1}}^{2} e^{2 \gamma \lambda^{-3}(l+1) \delta}+\frac{1}{\gamma^{4}}\left\|I^{\prime} g\right\|_{L^{2}}^{4} e^{2 \gamma \lambda^{-3}(l+1) \delta}\right) .
\end{aligned}
$$

Similarly, from Proposition 3.7 and 3.8, we have

$$
\begin{aligned}
& \left|\int_{0}^{(l+1) \delta}\left(\Lambda_{3}+\Lambda_{4}\right) e^{2 \gamma \lambda^{-3} t} d t\right| \\
\lesssim & \delta^{\frac{1}{6}}\left(N^{\prime}\right)^{-\frac{1}{2}+} \sum_{m=0}^{l}\left\|I^{\prime} v\right\|_{Y_{[m \delta,(m+1) \delta]}^{1}}^{3} e^{2 \gamma \lambda^{-3}(m+1) \delta} \\
\lesssim & \delta^{\frac{1}{6}}\left(C_{4} \lambda^{2} T^{\prime}\right)^{-1} \sum_{m=0}^{l}\left(\left\|I^{\prime} v(m \delta)\right\|_{H^{1}}^{3}+\left(\lambda^{-3}\left\|I^{\prime} g\right\|_{H^{1}}\right)^{3}\right) e^{2 \gamma \lambda^{-3}(m+1) \delta} .
\end{aligned}
$$

On one hand, (4.14) and (4.15) tell us that

$$
\begin{aligned}
& \left\|I^{\prime} v(m \delta)\right\|_{L^{2}}^{3} e^{2 \gamma \lambda^{-3}(m+1) \delta} \\
\lesssim & C_{1}^{2}\left(\left\|I^{\prime} v_{0}\right\|_{L^{2}}^{3}+\frac{1}{\gamma^{2}}\left\|I^{\prime} g\right\|_{L^{2}}^{3} e^{2 \gamma \lambda^{-3}(m+1) \delta}\right) \\
\lesssim & C_{1}^{2} \lambda^{2}\left(\left\|I^{\prime} v_{0}\right\|_{L^{2}}^{4}+\frac{1}{\gamma^{4}}\left\|I^{\prime} g\right\|_{L^{2}}^{4} e^{2 \gamma \lambda^{-3}(m+1) \delta}\right)
\end{aligned}
$$

and

$$
\begin{aligned}
& \left\|I^{\prime} v(m \delta)\right\|_{\dot{H}^{1}}^{3} e^{2 \gamma \lambda^{-3}(m+1) \delta} \\
& \lesssim\left(C_{1} C_{2}\right)^{\frac{1}{2}}\left(\left\|I^{\prime} v_{0}\right\|_{\dot{H}^{1}}^{2}+\left\|I^{\prime} v_{0}\right\|_{L^{2}}^{4}+\frac{1}{\gamma^{2}}\left\|I^{\prime} g\right\|_{\dot{H}^{1}}^{2} e^{2 \gamma \lambda^{-3}(m+1) \delta}\right. \\
& \left.\quad+\frac{1}{\gamma^{4}}\left\|I^{\prime} g\right\|_{L^{2}}^{4} e^{2 \gamma \lambda^{-3}(m+1) \delta}\right),
\end{aligned}
$$

on the other hand, we have

$$
\left(\lambda^{-3}\left\|I^{\prime} g\right\|_{H^{1}}\right)^{3} e^{2 \gamma \lambda^{-3}(m+1) \delta}
$$




$$
\begin{aligned}
& \lesssim \frac{1}{\gamma^{3}}\left\|I^{\prime} g\right\|_{L^{2}}^{3} e^{2 \gamma \lambda^{-3}(m+1) \delta}+\frac{1}{\gamma^{3}}\left\|I^{\prime} g\right\|_{\dot{H}^{1}}^{3} e^{2 \gamma \lambda^{-3}(m+1) \delta} \\
& \lesssim \lambda^{2} \frac{1}{\gamma^{4}}\left\|I^{\prime} g\right\|_{L^{2}}^{4} e^{2 \gamma \lambda^{-3}(m+1) \delta}+\left(C_{2}\right)^{\frac{1}{2}} \frac{1}{\gamma^{2}}\left\|I^{\prime} g\right\|_{\dot{H}^{1}}^{2} e^{2 \gamma \lambda^{-3}(m+1) \delta} \\
& \lesssim \lambda^{2}\left(\frac{1}{\gamma^{4}}\left\|I^{\prime} g\right\|_{L^{2}}^{4} e^{2 \gamma \lambda^{-3}(m+1) \delta}+\frac{1}{\gamma^{2}}\left\|I^{\prime} g\right\|_{\dot{H}^{1}}^{2} e^{2 \gamma \lambda^{-3}(m+1) \delta}\right) .
\end{aligned}
$$

Collecting (4.22)-(4.25), one gets

$$
\begin{aligned}
& \quad\left|\int_{0}^{(l+1) \delta}\left(\Lambda_{3}+\Lambda_{4}\right) e^{2 \gamma \lambda^{-3} t} d t\right| \\
& \lesssim \delta^{\frac{1}{6}}\left(C_{4} \lambda^{2} T^{\prime}\right)^{-1} C_{1}^{2} \lambda^{2}(l+1)\left(\left\|I^{\prime} v_{0}\right\|_{\dot{H}^{1}}^{2}+\left\|I^{\prime} v_{0}\right\|_{L^{2}}^{4}\right. \\
& \left.\quad+\frac{1}{\gamma^{2}}\left\|I^{\prime} g\right\|_{\dot{H}^{1}}^{2} e^{2 \gamma \lambda^{-3}(l+1) \delta}+\frac{1}{\gamma^{4}}\left\|I^{\prime} g\right\|_{L^{2}}^{4} e^{2 \gamma \lambda^{-3}(l+1) \delta}\right) \\
& \lesssim \delta^{-\frac{5}{6}} C_{1}^{2} C_{4}^{-1}\left(\left\|I^{\prime} v_{0}\right\|_{\dot{H}^{1}}^{2}+\left\|I^{\prime} v_{0}\right\|_{L^{2}}^{4}\right. \\
& \left.\quad+\frac{1}{\gamma^{2}}\left\|I^{\prime} g\right\|_{\dot{H}^{1}}^{2} e^{2 \gamma \lambda^{-3}(l+1) \delta}+\frac{1}{\gamma^{4}}\left\|I^{\prime} g\right\|_{L^{2}}^{4} e^{2 \gamma \lambda^{-3}(l+1) \delta}\right)
\end{aligned}
$$

which gives (4.21) by taking $C_{4}$ sufficiently large.

Proposition 4.4. Let $C_{2} \ll 1, C_{3}>1, C_{4} \gg 1$ and $T>0$ be given. Assume $u$ is a solution of (1.4) on $[0, T]$. If $N^{\frac{3}{11}-} \geqslant \gamma, N^{0+} \geqslant C_{4} T$,

$$
\left\|I u_{0}\right\|_{L^{2}}^{2}+\frac{1}{\gamma^{2}}\|I f\|_{L^{2}}^{2} e^{2 \gamma T} \leqslant C_{2} N^{\frac{2}{11}-}
$$

and

$$
\left\|I u_{0}\right\|_{\dot{H}^{1}}^{2}+\frac{1}{\gamma^{2}}\|I f\|_{\dot{H}^{1}}^{2} e^{2 \gamma T} \leqslant C_{2} N^{\frac{2}{11}-},
$$

then we have

$$
\begin{gathered}
\|I u(T)\|_{L^{2}}^{2} e^{2 \gamma T} \leqslant C_{3}\left(\left\|I u_{0}\right\|_{L^{2}}^{2}+\frac{1}{\gamma^{2}}\|I f\|_{L^{2}}^{2} e^{2 \gamma T}\right), \\
\|I u(T)\|_{\dot{H}^{1}}^{2} e^{2 \gamma T} \leqslant C_{3}\left(\left\|I u_{0}\right\|_{\dot{H}^{1}}^{2}+\left\|I u_{0}\right\|_{L^{2}}^{4}+\frac{1}{\gamma^{2}}\|I f\|_{\dot{H}^{1}}^{2} e^{2 \gamma T}+\frac{1}{\gamma^{4}}\|I f\|_{L^{2}}^{4} e^{2 \gamma T}\right) .
\end{gathered}
$$

Proof. Notice that $\left\|I^{\prime} v\right\|_{L^{2}}^{2}=\lambda^{-2}\|I u\|_{L^{2}}^{2}, \mid I^{\prime} g\left\|_{L^{2}}^{2}=\lambda^{-2}\right\| I f\left\|_{L^{2}}^{2},\right\| I^{\prime} v\left\|_{\dot{H}^{1}}^{2}=\lambda^{-4}\right\| I u \|_{\dot{H}^{1}}^{2}$, $\left\|I^{\prime} g\right\|_{\dot{H}^{1}}^{2}=\lambda^{-4}\|I f\|_{\dot{H}^{1}}^{2}$ and the fact $\lambda^{2}\left\|I^{\prime} v_{0}\right\|_{L^{2}}^{2}=\left\|I u_{0}\right\|_{L^{2}}^{2}>\frac{1}{2}\left\|u_{0}\right\|_{L^{2}}^{2}$ if $N$ is sufficiently large, we acquire Proposition 4.4 via taking $\lambda=N^{\frac{1}{11}-}, N^{\prime}=\frac{N}{\lambda}$ and $T^{\prime}=\lambda^{3} T$ in Proposition 4.3.

Finally, we show the exsitence of the global attractor.

Proof of Theorem 1.2. Let $0<\epsilon \ll 1$ be fixed. We choose $T_{1}>0$ so that

$$
e^{2 \gamma T_{1}}>\left(\left\|u_{0}\right\|_{H^{s}}^{2}+\left\|u_{0}\right\|_{L^{2}}^{4}\right)\left(\frac{1}{\gamma^{2}}\|f\|_{H^{1}}^{2}+\frac{1}{\gamma^{4}}\|f\|_{L^{2}}^{4}\right)^{-1} \max \left\{\gamma^{\frac{22(1-s)}{3}+},\left(C_{4} T_{1}\right)^{\frac{2(1-s)}{\epsilon}},\right.
$$




$$
\left.\left(\frac{C_{2}}{2}\left\|u_{0}\right\|_{H^{s}}^{-2}\right)^{\frac{11(1-s)}{(10-11 s)}-},\left(2 C_{2}^{-1} \gamma^{-2}\|f\|_{H^{1}}^{2} e^{2 \gamma T_{1}}\right)^{11(1-s)+}\right\}
$$

which is possible as $11(1-s)<1$. $T_{1}$ depends only on $\left\|u_{0}\right\|_{H^{s}},\|f\|_{H^{1}}$ and $\gamma$. Set

$$
N=\max \left\{\gamma^{\frac{11}{3}+},\left(C_{4} T_{1}\right)^{\frac{1}{\epsilon}},\left(\frac{C_{2}}{2}\left\|u_{0}\right\|_{H^{s}}^{-2}\right)^{\frac{11}{2(10-11 s)}-},\left(2 C_{2}^{-1} \gamma^{-2}\|f\|_{H^{1}}^{2} e^{2 \gamma T_{1}}\right)^{\frac{11}{2}+}\right\} .
$$

From the choice of $T_{1}$ and $N$, we know

$$
N^{\frac{3}{11}-} \geqslant \gamma, \quad N^{\epsilon} \geqslant C_{4} T_{1}
$$

and

$$
\begin{gathered}
\left\|I u_{0}\right\|_{H^{1}}^{2} \leqslant N^{2-2 s}\left\|u_{0}\right\|_{H^{s}}^{2} \leqslant \frac{C_{2}}{2} N^{\frac{2}{11}-}, \\
\gamma^{-2}\|I f\|_{H^{1}}^{2} e^{2 \gamma T_{1}} \leqslant \frac{C_{2}}{2} N^{\frac{2}{11}-} .
\end{gathered}
$$

Hence, from Proposition 4.4, one gains

$$
\begin{aligned}
\left\|u\left(T_{1}\right)\right\|_{H^{s}}^{2} & \leqslant\left\|I u\left(T_{1}\right)\right\|_{H^{1}}^{2} \\
& \leqslant C_{3}\left(\left\|I u_{0}\right\|_{H^{1}}^{2} e^{-2 \gamma T}+\left\|I u_{0}\right\|_{L^{2}}^{4} e^{-2 \gamma T}+\frac{1}{\gamma^{2}}\|I f\|_{H^{1}}^{2}+\frac{1}{\gamma^{4}}\|I f\|_{L^{2}}^{4}\right) \\
& \leqslant C_{3}\left(N^{2(1-s)}\left\|u_{0}\right\|_{H^{s}}^{2} e^{-2 \gamma T}+\left\|u_{0}\right\|_{L^{2}}^{4} e^{-2 \gamma T}+\frac{1}{\gamma^{2}}\|f\|_{H^{1}}^{2}+\frac{1}{\gamma^{4}}\|f\|_{L^{2}}^{4}\right) .
\end{aligned}
$$

From (4.26) and (4.27), we get

$$
N^{2(1-s)} e^{-2 \gamma T_{1}}\left(\left\|u_{0}\right\|_{H^{s}}^{2}+\left\|u_{0}\right\|_{L^{2}}^{4}\right)<\frac{1}{\gamma^{2}}\|f\|_{H^{1}}^{2}+\frac{1}{\gamma^{4}}\|f\|_{L^{2}}^{4}
$$

which helps us give the bound

$$
\left\|u\left(T_{1}\right)\right\|_{H^{s}}^{2} \leqslant 2 C_{3}\left(\frac{1}{\gamma^{2}}\|f\|_{H^{1}}^{2}+\frac{1}{\gamma^{4}}\|f\|_{L^{2}}^{4}\right)<K_{1}
$$

where $K_{1}$ depends only on $\|f\|_{H^{1}}$ and $\gamma$.

In the next place, one can fix $T_{2}>0$ and solve (1.4) on time interval $\left[T_{1}, T_{1}+T_{2}\right]$ with initial data replaced by $u\left(T_{1}\right)$. Let $K_{2}>0$ be sufficiently large such that

$$
\begin{gathered}
K_{2} e^{2 \gamma t}>\max \left\{\gamma^{\frac{22(1-s)}{3}+},\left(C_{4} t\right)^{\frac{2(1-s)}{\epsilon}},\left(2 C_{2}^{-1} K_{1}\right)^{\frac{11(1-s)}{(11 s-10)}+},\right. \\
\left.\left(2 C_{2}^{-1} \gamma^{-2}\|f\|_{H^{1}}^{2} e^{2 \gamma t}\right)^{11(1-s)+}\right\}
\end{gathered}
$$


for any $t>0$. Set $N^{2(1-s)}=K_{2} e^{2 \gamma T_{2}}$, then inequality (4.28) verifies the assumptions in Proposition 4.4 and hence we obtain

$$
\begin{aligned}
\left\|I u\left(T_{1}+T_{2}\right)\right\|_{H^{1}}^{2} & \leqslant C_{3}\left(N^{2(1-s)}\left\|u\left(T_{1}\right)\right\|_{H^{s}}^{2} e^{-2 \gamma T_{2}}+\left\|u\left(T_{1}\right)\right\|_{L^{2}}^{4} e^{-2 \gamma T_{2}}+\frac{1}{\gamma^{2}}\|f\|_{H^{1}}^{2}+\frac{1}{\gamma^{4}}\|f\|_{L^{2}}^{4}\right) \\
& \leqslant C_{3}\left(K_{1} K_{2}+K_{1}^{2}+\frac{1}{\gamma^{2}}\|f\|_{H^{1}}^{2}+\frac{1}{\gamma^{4}}\|f\|_{L^{2}}^{4}\right)<K_{3} .
\end{aligned}
$$

For $t>T_{1}$, we define the maps $M_{1}(t)$ and $M_{2}(t)$ as

$$
\widehat{M_{1}(t) u_{0}}=\left.\widehat{A(t) u_{0}}\right|_{|\zeta|<N_{t}}, \quad \widehat{M_{2}(t) u_{0}}=\left.\widehat{A(t) u_{0}}\right|_{|\zeta|>N_{t}},
$$

where $A(t) u_{0}=u(t)$ and $N_{t}=\left(K_{2} e^{2 \gamma\left(t-T_{1}\right)}\right)^{\frac{1}{2(1-s)}}$.

It's easy to see that for $t>T_{1}$,

$$
\begin{gathered}
\left\|M_{1}(t) u_{0}\right\|_{H^{1}}^{2} \leqslant\|I u(t)\|_{H^{1}}^{2}<K_{3}, \\
\left\|M_{2}(t) u_{0}\right\|_{H^{s}}^{2} \leqslant N^{2 s-2}\|I u(t)\|_{H^{1}}^{2}<K_{2}^{-1} K_{3} e^{-2 \gamma\left(t-T_{1}\right)} .
\end{gathered}
$$

Hence we obtain Theorem 1.2 by taking $K=\max \left\{K_{3}^{\frac{1}{2}}, K_{2}^{-\frac{1}{2}} K_{3}^{\frac{1}{2}}\right\}$.

\section{Acknowledgment}

The author would like to express his deep gratitude to Professor Yoshio Tsutsumi and Professor Baoxiang Wang for their contructive guidance and kind help.

\section{References}

[1] T. R. Akylas. On the excitation of long nonlinear water waves by a moving pressure distribution. J. Fluid Mech. 141 (1984), 455-466.

[2] J. Bourgain. Exponential sums and nonlinear Schrdinger equations. Geom. Funct. Anal., 3(2) (1993), 157-178.

[3] J. Colliander, M. Keel, G. Staffilani, H. Takaoka, and T. Tao. Almost conservation laws and global rough solutions to a Nonlinear Schrödinger equation. Math. Res. Lett., 9(5-6) (2002), 659-682.

[4] J. Colliander, M. Keel, G. Staffilani, H. Takaoka, and T. Tao. Sharp Global well-posedness for KdV and modified KdV on $\mathbb{R}$ and T. J. Amer. Math. Soc., 16 (2003), 705-749.

[5] J. Colliander, M. Keel, G. Staffilani, H. Takaoka, and T. Tao. Resonant decompositions and the $I$-method for the cubic nonlinear Schrödinger equation on $\mathbb{R}^{2}$. Discrete Contin. Dyn. Syst. 21(3) (2008), 665-686.

[6] Andrei V. Faminskii. The Cauchy problem for the Zakharov-Kuznetsov equation. Differ. Equations, 31(6) (1995), 1002-1012.

[7] Jean Ginibre, Yoshio Tsutsumi, and Giorgio Velo. On the Cauchy problem for the Zakharov system. J. Funct. Anal., 151(2) (1997), 384-436. 
[8] O. Goubet. Regularity of the attractor for a weakly damped nonlinear Schrödinger equation in $\mathbb{R}^{2}$. Adv. Differential Equations 3(3) (1998), 337-360.

[9] Axel Grünrock and Sebastian Herr. The Fourier restriction norm method for the ZakharovKuznetsov equation. Discrete Contin. Dyn. Syst., 34(5) (2014), 2061-2068.

[10] M. Hadac, S. Herr, and H. Koch. Well-posedness and scattering for the KP-II equation in a critical space, Ann. Inst. H. Poincaré Anal. Non Linéaire, 26 (2009), 917-941.

[11] A. Haraux. Two remarks on dissipative hyperbolic problems. Nonlinear partial differential equations and their applications. Collge de France seminar, Vol. VII (Paris, 1983-1984), 6, 161-179, Res. Notes in Math., 122, Pitman, Boston, MA, 1985.

[12] Carlos E. Kenig, Gustavo Ponce, and Luis Vega. Well-posedness and scattering results for the generalized Korteweg-de Vries equation via the contraction principle. Comm. Pure Appl. Math., 46(4) (1993), 527-620.

[13] H. Koch and D. Tataru. Dispersive estimates for principlally normal pseudo-differential operators. Comm. Pure Appl. Math., 58 (2005), 217-284.

[14] H. Koch and D. Tataru. A priori bounds for the 1D cubic NLS in negative Sobolev spaces. Int. Math. Res. Not., 16 (2007), 36-71.

[15] H. Koch and D. Tataru. Energy and local energy bounds for the 1D cubic NLS equation in $H^{1 / 4}$. Ann. Inst. H. Poincaré Anal. Non Linéaire, 29 (2012), 955-988.

[16] E.A. Kuznetsov and V.E. Zakharov. On the three dimensional solitons. Sov. Phys. JETP, 39 (1974), $285-286$.

[17] Ernst W. Laedke and Karl-Heinz Spatschek. Nonlinear ion-acoustic waves in weak magnetic fields. Phy. Fluids, 25(6) (1982), 985-989.

[18] David Lannes, Felipe Linares, and Jean-Claude Saut. The Cauchy problem for the Euler-Poisson system and derivation of the Zakharov-Kuznetsov equation. Studies in phase space analysis with applications to PDEs, Springer New York (2013), 181-213.

[19] Felipe Linares and Ademir Pastor. Well-posedness for the two-dimensional modified ZakharovKuznetsov equation. SIAM J. Math. Anal., 41(4) (2009), 1323-1339.

[20] I. Moise and R. Rosa. On the regularity of the global attractor of a weakly damped, forced Kortewegde Vries equation. Adv. Differential Equations, 2(2) (1997), 257-296.

[21] Luc Molinet and Didier Pilod. Bilinear Strichartz estimates for the Zakharov-Kuznetsov equation and applications. Ann. Inst. H. Poincaré Anal. Non Linéaire, 32(2) (2015), 347-371.

[22] Luc Molinet and Stéphane Vento. Improvement of the energy method for strongly nonresonant dispersive equations and applications. Anal. PDE, 8(6) (2015), 1455-1495.

[23] E. Ott and R. N. Sudan. Damping of Solitary Waves. Physics of Fluids, 13 (1970), 1432-1434.

[24] G. Prashant. Existence of the global attractor for weakly damped, forced mKdV equation below energy spaces. Preprint, 2018.

[25] M. J. Shan. Well-Posedness for the Two-dimensional Zakharov-Kuznetsov Equation. arXiv:1807.10123 2 [math.AP].

[26] R. Temam. Infinite-Dimensional Dynamical Systems in Mechanics and Physics. Springer Verlag, Second Edition, 1997.

[27] K. Tsugawa. Existence of the global attractor for weakly damped, forced KdV equation on Sobolev spaces of negative index. Communications on Pure and Applied Analysis, 3(2) (2004), 301-318. 
[28] B. X. Wang, Z. H. Huo, C. C. Hao and Z. H. Guo. Harmonic Analysis Method for Nonlinear Evolution Equations. I. World Scientific Publishing Co., Pte. Ltd., Hackensack, NJ (2011).

[29] T. Y. Wu. Generation of upstream advancing solitons by moving disturbances. Journal of Fluid Mechanics, 184 (1987), 75-99.

[30] X. Yang. Global attractor for the weakly damped forced KdV equation in Sobolev spaces of low regularity. Nonlinear Differential Equations and Applications, 18(3) (2011), 273-285. 